\title{
CONDITIONAL STABILITY AND CONVERGENCE OF A FULLY DISCRETE SCHEME FOR THREE-DIMENSIONAL NAVIER-STOKES EQUATIONS WITH MASS DIFFUSION*
}

\author{
F. GUILLÉN-GONZÁLEZ† AND J. V. GUTIÉRREZ-SANTACREU†
}

\begin{abstract}
We construct a fully discrete numerical scheme for three-dimensional incompressible fluids with mass diffusion (in density-velocity-pressure formulation), also called the KazhikhovSmagulov model. We will prove conditional stability and convergence, by using at most $C^{0}$-finite elements, although the density of the limit problem will have $H^{2}$-regularity. The key idea of our argument is first to obtain pointwise estimates for the discrete density by imposing the constraint $\lim _{(h, k) \rightarrow 0} h / k=0$ on the time and space parameters $(k, h)$. Afterwards, under the same constraint on the parameters, strong estimates for the discrete density in $l^{\infty}\left(H^{1}\right)$ and for the discrete Laplacian of the density in $l^{2}\left(L^{2}\right)$ are obtained. From here, the compactness and convergence of the scheme can be concluded with similar arguments as we used in [Math. Comp., to appear], where a different scheme is studied for two-dimensional domains which is unconditionally stable and convergent. Moreover, we study the asymptotic behavior of the numerical scheme as the diffusion parameter $\lambda$ goes to zero, obtaining convergence as $(k, h, \lambda) \rightarrow 0$ towards a weak solution of the density-dependent Navier-Stokes system provided that the constraint $\lim _{(\lambda, h, k) \rightarrow 0} h /\left(\lambda^{2} k\right)=0$ on $(h, k, \lambda)$ is satisfied.
\end{abstract}

Key words. three-dimensional Kazhikhov-Smagulov models, density-dependent Navier-Stokes equations, finite elements, stability, convergence

AMS subject classifications. 35Q35, 65M12, 65M60

DOI. $10.1137 / 07067951 \mathrm{X}$

\section{Introduction.}

1.1. The model. Let $\Omega \subset \mathbb{R}^{3}$ be an open bounded set with boundary $\Gamma$. We denote by $[0, T](0<T<+\infty)$ the time interval of observation. We will use the notation $Q=\Omega \times(0, T), \Sigma=\Gamma \times(0, T)$, and $\boldsymbol{n}(\boldsymbol{x})$ the outwards unit normal vector to $\Gamma$ at the point $\boldsymbol{x} \in \Gamma$.

We consider the Navier-Stokes system with mass diffusion (the so-called Kazhikhov-Smagulov model) in $Q$ :

$$
\left\{\begin{aligned}
\rho \boldsymbol{u}_{t}+((\rho \boldsymbol{u}-\lambda \nabla \rho) \cdot \nabla) \boldsymbol{u}-\nabla \cdot\left(\mu \nabla \boldsymbol{u}-\lambda \rho(\nabla \boldsymbol{u})^{t}\right)+\nabla p & =\rho \boldsymbol{f} \\
\nabla \cdot \boldsymbol{u}=0, \quad \rho_{t}+\boldsymbol{u} \cdot \nabla \rho-\lambda \Delta \rho & =0 .
\end{aligned}\right.
$$

The unknowns for this model are $\rho: Q \rightarrow \mathbb{R}^{+}$, the density of the fluid, $\boldsymbol{u}: Q \rightarrow \mathbb{R}^{3}$, the incompressible (averaged) velocity vector field, and $p: Q \rightarrow \mathbb{R}$, a potential function (modified pressure).

Model (1.1) can be derived from the compressible Navier-Stokes system, by imposing that the compressible velocity $\boldsymbol{v}$ can be decomposed as $\boldsymbol{v}=\boldsymbol{u}-\lambda \nabla \log \rho$, with $\nabla \cdot \boldsymbol{u}=0$ (it decomposes into an incompressible part $\boldsymbol{u}$ and a potential part $-\lambda \nabla \log \rho$ ), and eliminating $\lambda^{2}$-terms (see [11]).

\footnotetext{
${ }^{*}$ Received by the editors January 9, 2007; accepted for publication (in revised form) January 22, 2008; published electronically May 9, 2008. This work was partially supported by the Spanish project BFM2003-06446-C02-01.

http://www.siam.org/journals/sinum/46-5/67951.html

${ }^{\dagger}$ Departamento E.D.A.N., University of Sevilla, Aptdo. 1160, 41080 Sevilla, Spain (guillen@us.es, juanvi@us.es).
} 
We complete (1.1) with the boundary conditions on $\Sigma$ :

$$
\boldsymbol{u}_{\left.\right|_{\Sigma}}=0,\left.\quad \frac{\partial \rho}{\partial \boldsymbol{n}}\right|_{\Sigma}=0
$$

and the initial conditions in $\Omega$ :

$$
\left.\rho\right|_{t=0}=\rho_{0},\left.\quad \boldsymbol{u}\right|_{t=0}=\boldsymbol{u}_{0}
$$

where $\rho_{0}: \Omega \rightarrow \mathbb{R}^{+}$and $\boldsymbol{u}_{0}: \Omega \rightarrow \mathbb{R}^{d}$ are given functions.

Throughout this work, we assume the hypothesis on the initial density:

$$
0<m \leq \rho_{0}(\boldsymbol{x}) \leq M \quad \text { in } \Omega .
$$

1.2. Known results. Concerning the simplified model (1.1), Kazhikhov and Smagulov [12] proved, via a semi-Galerkin method, the existence of global weak solutions, under the following hypothesis:

$$
\lambda<2 \mu /(M-m),
$$

and the existence of local strong solutions (which is global in the two-dimensional case). Salvi [14] proved the existence of weak solutions for noncylindrical domains. On the other hand, Secchi [16] studied the problem for $\Omega=\mathbb{R}^{3}$, proving the local existence and uniqueness of strong solutions by using a fixed point argument.

For the complete model (including the $\lambda^{2}$-terms), Beirão da Veiga [2] and Secchi [15] established the local existence of strong solutions by using linearization and a fixed point argument. In [15], global existence and uniqueness are shown for twodimensional (2D) domains by imposing that $\lambda / \mu$ is small enough as well as the asymptotic behavior as $\lambda \rightarrow 0$ towards a weak solution of the density-dependent Navier-Stokes problem:

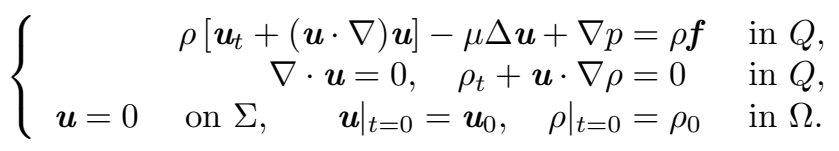

In the case of nonnegative initial density and 3D domains, Guillén-González [9] proved the global existence of weak solutions and the behavior, as $\lambda \rightarrow 0$, towards the density-dependent Navier-Stokes system (1.6). Recently, the existence and regularity of strong solutions have been proved in [10] by means of an iterative method (jointly with some error estimates).

A time-space numerical scheme has been recently developed by using $C^{0}$-finite elements for density and velocity in [11] for model (1.1) in 2D domains, which is unconditionally stable and convergent towards the (unique) weak solution of the continuous problem. This scheme is of the backward Euler type, where in each time step the computation of the density and the velocity pressure are decoupled, by means of linear problems.

Concerning the numerical analysis for the density-dependent Navier-Stokes problem, a stable and convergent scheme is proposed in [13], by using in particular a discontinuous Galerkin finite element method to approximate the density transport equation. 
1.3. Main results of the paper. Our main objective is to design a linear scheme by using finite elements to approximate all unknowns (density, velocity, and pressure) of problem (1.1)-(1.3). To this end, we consider for simplicity a uniform partition of $[0, T],\left(t_{n}=n k\right)_{n=0}^{n=N}$, with $k=T / N$ being the time step, and propose a backward Euler time scheme, implicit with respect to the diffusion terms and semiimplicit with respect to the convection terms. The finite element spaces must verify specific properties which we will describe in section 3.1.

In what follows we consider the notation $(\cdot, \cdot)$ and $|\cdot|$ for the $L^{2}(\Omega)$-inner product and the $L^{2}(\Omega)$-norm, respectively. Also, we denote that $\|u\|=|\nabla u|$, which is an equivalent norm to the usual one in $H_{0}^{1}(\Omega)$.

The scheme is described as follows:

Initialization: Let $\left(\boldsymbol{u}_{h}^{0}, \rho_{h}^{0}\right) \in \boldsymbol{V}_{h} \times W_{h}$ be an approximation of $\left(\boldsymbol{u}_{0}, \rho_{0}\right)$ as $h \rightarrow 0$.

Time step $n+1$ : Given $\left(\rho_{h}^{n}, \boldsymbol{u}_{h}^{n}, p_{h}^{n}\right) \in W_{h} \times \boldsymbol{V}_{h} \times M_{h}$,

1. find $\left(\boldsymbol{w}_{h}^{n}, q_{h}^{n}\right) \in \widetilde{\boldsymbol{V}}_{h} \times \widetilde{M}_{h}$ such that, for each $\left(\overline{\boldsymbol{w}}_{h}, \bar{q}_{h}\right) \in \widetilde{\boldsymbol{V}}_{h} \times \widetilde{M}_{h}$,

$$
\left(\nabla \boldsymbol{w}_{h}^{n}, \nabla \overline{\boldsymbol{w}}_{h}\right)-\left(q_{h}^{n}, \nabla \cdot \overline{\boldsymbol{w}}_{h}\right)=\left(\nabla \boldsymbol{u}_{h}^{n}, \nabla \overline{\boldsymbol{w}}_{h}\right), \quad\left(\nabla \cdot \boldsymbol{w}_{h}^{n}, \bar{q}_{h}\right)=0
$$

2. find $\rho_{h}^{n+1} \in W_{h}$ such that, for each $\bar{\rho}_{h} \in W_{h}$,

$$
\left(\frac{\rho_{h}^{n+1}-\rho_{h}^{n}}{k}, \bar{\rho}_{h}\right)+\left(\boldsymbol{w}_{h}^{n} \cdot \nabla \rho_{h}^{n+1}, \bar{\rho}_{h}\right)+\lambda\left(\nabla \rho_{h}^{n+1}, \nabla \bar{\rho}_{h}\right)=0
$$

3. find $\left(\boldsymbol{u}_{h}^{n+1}, p_{h}^{n+1}\right) \in \boldsymbol{V}_{h} \times M_{h}$ such that, for each $\left(\overline{\boldsymbol{u}}_{h}, \bar{p}_{h}\right) \in \boldsymbol{V}_{h} \times M_{h}$,

$$
\begin{aligned}
& \left\{\begin{array}{c}
\left(\rho_{h}^{n} \frac{\boldsymbol{u}_{h}^{n+1}-\boldsymbol{u}_{h}^{n}}{k}, \overline{\boldsymbol{u}}_{h}\right)+\frac{1}{2}\left(\frac{\rho_{h}^{n+1}-\rho_{h}^{n}}{k} \boldsymbol{u}_{h}^{n+1}, \overline{\boldsymbol{u}}_{h}\right)+a\left(\rho_{h}^{n+1}, \boldsymbol{u}_{h}^{n+1}, \overline{\boldsymbol{u}}_{h}\right) \\
+c\left(\rho_{h}^{n+1} \boldsymbol{u}_{h}^{n}-\lambda \nabla \rho_{h}^{n+1}, \boldsymbol{u}_{h}^{n+1}, \overline{\boldsymbol{u}}_{h}\right)=\left(\rho_{h}^{n+1} \boldsymbol{f}^{n+1}, \overline{\boldsymbol{u}}_{h}\right)+\left(p_{h}^{n+1}, \nabla \cdot \overline{\boldsymbol{u}}_{h}\right),
\end{array}\right. \\
& \left(\begin{array}{l}
(1.9) \\
(1.10)
\end{array}\right. \\
& \left(\nabla \cdot \boldsymbol{u}_{h}^{n+1}, \bar{p}_{h}\right)=0,
\end{aligned}
$$

where

$$
\begin{gathered}
\boldsymbol{f}^{n+1}=\frac{1}{k} \int_{t_{n}}^{t_{n+1}} \boldsymbol{f}(t) d t \\
a(\rho, \boldsymbol{u}, \boldsymbol{v})=\mu(\nabla \boldsymbol{u}, \nabla \boldsymbol{v})+\lambda \int_{\Omega}\left(\frac{\widetilde{M}+\widetilde{m}}{2}-\rho\right)(\nabla \boldsymbol{u})^{t}: \nabla \boldsymbol{v} d \boldsymbol{x},
\end{gathered}
$$

with $\widetilde{M}>M, 0<\widetilde{m}<m$ such that $\lambda \frac{\widetilde{M}-\widetilde{m}}{2}<\mu$ (here (1.5) is imposed), and

$$
c(\boldsymbol{w}, \boldsymbol{u}, \boldsymbol{v})=\frac{1}{2}[((\boldsymbol{w} \cdot \nabla) \boldsymbol{u}, \boldsymbol{v})-((\boldsymbol{w} \cdot \nabla) \boldsymbol{v}, \boldsymbol{u})] .
$$

The following properties of continuity and coercivity hold:

$$
\begin{aligned}
a(\rho, \boldsymbol{u}, \boldsymbol{u}) \geq & \frac{\mu_{1}}{2}\|\boldsymbol{u}\|^{2} \text { if } \widetilde{m} \leq \rho \leq \widetilde{M}, \quad \text { with } \frac{\mu_{1}}{2}=\mu-\lambda \frac{\widetilde{M}-\widetilde{m}}{2}(>0), \\
& a(\rho, \boldsymbol{u}, \boldsymbol{v}) \leq C_{\lambda}\|\boldsymbol{u}\|\|\boldsymbol{v}\| \quad\left(\text { if }\|\rho\|_{L^{\infty}(\Omega)} \leq C\right), \\
& c(\boldsymbol{w}, \boldsymbol{u}, \boldsymbol{u})=0, \quad c(\boldsymbol{w}, \boldsymbol{u}, \boldsymbol{v}) \leq C\|\boldsymbol{w}\|_{L^{3}}\|\boldsymbol{u}\|\|\boldsymbol{v}\| .
\end{aligned}
$$

Copyright $@$ by SIAM. Unauthorized reproduction of this article is prohibited. 
Here and in what follows, we denote by $C_{\lambda}$ and $C$ different positive constants independent of $(h, k)$ and $(h, k, \lambda)$, respectively.

From the computational point of view, scheme (1.7)-(1.10) decouples density $\rho_{h}^{n+1}$ and velocity pressure $\left(\boldsymbol{u}_{h}^{n+1}, p_{h}^{n+1}\right)$, whereas $\boldsymbol{w}_{h}^{n}$ is an intermediate velocity obtained as the $H^{1}$ orthogonal projection of $\boldsymbol{u}_{h}^{n}$ onto a discrete free-divergence space. We will see that scheme (1.7)-(1.10) is conditionally stable and convergent. As in many practical situations, the diffusion parameter is small; we will prove that, when the diffusion parameter $\lambda$ and the space and time parameters $(h, k)$ goes to zero, scheme (1.7)-(1.10) approximates to a weak solution of the density-dependent Navier-Stokes system (1.6), under a constraint involving the parameters $h, k$, and $\lambda$. In fact, to our knowledge, it is the first convergent scheme to (1.6) based on $C^{0}$-finite elements for the discrete density, avoiding to perform directly an algorithm for (1.6) which presents important difficulties by itself, mainly for the approximation of the density transport equation. Recall that in [13] a convergent scheme for (1.6) is given based on a discontinuous Galerkin method for the density.

The corresponding study for the complete model, with $\lambda^{2}$-terms, will be the subject of a forthcoming paper.

By defining in $[0, T]$ piecewise constant functions $\boldsymbol{u}_{h, k}, \rho_{h, k}$ such that $\boldsymbol{u}_{h, k}$, $\left.\rho_{h, k}\right|_{\left(t_{n-1}, t_{n}\right]}=\boldsymbol{u}_{h}^{n}, \rho_{h}^{n}$, respectively, that we will denote by $\boldsymbol{u}_{h, k, \lambda}, \rho_{h, k, \lambda}$ when the case of diffusion parameter $\lambda \rightarrow 0$ is considered, we present the following main results of this paper.

THEOREM 1.1. Assume hypotheses (H0)-(H5) given in section 3.1 . If the constraint on the parameters

$$
h / k \rightarrow 0 \quad \text { as }(h, k) \rightarrow 0
$$

holds, then there exists a convergent subsequence of $\left(\boldsymbol{u}_{h, k}, \rho_{h, k}\right)$ (denoted in the same way) as $(h, k) \rightarrow 0$ towards a weak solution $(\boldsymbol{u}, \rho)$ of problem (1.1)-(1.3) (see Definition $2.1)$, in the following sense: $\left(\boldsymbol{u}_{h, k}, \rho_{h, k}\right) \rightarrow(\boldsymbol{u}, \rho)$ in $L^{2}\left(0, T ; \boldsymbol{L}^{2}(\Omega)\right) \times L^{2}\left(0, T ; H^{1}(\Omega)\right)$ strong, in $L^{\infty}\left(0, T ; \boldsymbol{L}^{2}(\Omega)\right) \times\left(H^{1}(\Omega) \cap L^{\infty}(\Omega)\right)$-weak $\star$, and in $L^{2}\left(0, T ; \boldsymbol{H}_{0}^{1}(\Omega)\right) \times$ $L^{4}\left(0, T ; W^{1,3}(\Omega)\right)$-weak. Moreover, $\widetilde{m} \leq \rho_{h, k} \leq \widetilde{M}$.

THEOREM 1.2. Under the hypotheses of Theorem 1.1 and by extending (H2) by $\left(\mathrm{H} 2^{\prime}\right)$ (given in section 8.1) and changing $(\mathrm{S})$ by the more restrictive constraint

$$
h /\left(k \lambda^{2}\right) \rightarrow 0 \quad \text { as }(\lambda, h, k) \rightarrow 0,
$$

then there exists a convergent subsequence of $\left(\boldsymbol{u}_{h, k, \lambda}, \rho_{h, k, \lambda}\right)$ as $(h, k, \lambda) \rightarrow 0$ towards a weak solution $(\boldsymbol{u}, \rho)$ of the density-dependent Navier-Stokes problem (1.6) (see Definition 8.1) in the following sense: $\boldsymbol{u}_{h, k, \lambda} \rightarrow \boldsymbol{u}$ in $L^{2}\left(0, T ; \boldsymbol{L}^{2}(\Omega)\right)$-strong, in $L^{\infty}\left(0, T ; \boldsymbol{L}^{2}(\Omega)\right)$-weak , and in $L^{2}\left(0, T ; \boldsymbol{H}_{0}^{1}(\Omega)\right)$-weak, and $\rho_{h, k, \lambda} \rightarrow \rho$ in $L^{\infty}(Q)$ weak*.

The main ideas for the derivation of this scheme can be found in [11], where the following scheme has been studied:

Time step $(n+1)$ : Given $\left(\rho_{h}^{n}, \boldsymbol{u}_{h}^{n}, p_{h}^{n}\right) \in W_{h} \times \boldsymbol{V}_{h} \times M_{h}$,

1. find $\rho_{h}^{n+1} \in W_{h}$ such that, for each $\bar{\rho}_{h} \in W_{h}$,

$$
\left(\frac{\rho_{h}^{n+1}-\rho_{h}^{n}}{k}, \bar{\rho}_{h}\right)+\lambda\left(\nabla \rho_{h}^{n+1}, \nabla \bar{\rho}_{h}\right)=-\left(\boldsymbol{u}_{h}^{n} \cdot \nabla \rho_{h}^{n}, \bar{\rho}_{h}\right)
$$

Copyright (c) by SIAM. Unauthorized reproduction of this article is prohibited. 
2. find $\left(\boldsymbol{u}_{h}^{n+1}, p_{h}^{n+1}\right) \in \boldsymbol{V}_{h} \times M_{h}$ such that, for each $\left(\overline{\boldsymbol{u}}_{h}, \bar{p}_{h}\right) \in \boldsymbol{V}_{h} \times M_{h}$,

$$
\begin{gathered}
\left(\left[\rho_{h}^{n}\right]_{T} \frac{\boldsymbol{u}_{h}^{n+1}-\boldsymbol{u}_{h}^{n}}{k}, \overline{\boldsymbol{u}}_{h}\right)+\frac{1}{2}\left(\frac{\left[\rho_{h}^{n+1}\right]_{T}-\left[\rho_{h}^{n}\right]_{T}}{k}, \boldsymbol{u}_{h}^{n+1} \cdot \overline{\boldsymbol{u}}_{h}\right) \\
+a\left(\left[\rho_{h}^{n+1}\right]_{T}, \boldsymbol{u}_{h}^{n+1}, \overline{\boldsymbol{u}}_{h}\right)+c\left(\rho_{h}^{n+1} \boldsymbol{u}_{h}^{n}-\lambda \nabla \rho_{h}^{n+1}, \boldsymbol{u}_{h}^{n+1}, \overline{\boldsymbol{u}}_{h}\right) \\
-\left(p_{h}^{n+1}, \nabla \cdot \overline{\boldsymbol{u}}_{h}\right)=\left(\left[\rho_{h}^{n+1}\right]_{T} \boldsymbol{f}^{n+1}, \overline{\boldsymbol{u}}_{h}\right) \\
\left(\nabla \cdot \boldsymbol{u}_{h}^{n+1}, \bar{p}_{h}\right)=0
\end{gathered}
$$

where

$$
\left[w_{h}\right]_{T}\left(\boldsymbol{x}_{i}\right)=\left\{\begin{array}{lll}
w_{h}\left(\boldsymbol{x}_{i}\right) & \text { if } & w_{h}\left(\boldsymbol{x}_{i}\right) \in[m, M] \\
m & \text { if } \quad w_{h}\left(\boldsymbol{x}_{i}\right)<m, \\
M & \text { if } \quad w_{h}\left(\boldsymbol{x}_{i}\right)>M
\end{array}\right.
$$

with $\boldsymbol{x}_{i}$ the nodes of the mesh $\mathcal{T}_{h}$ of $\Omega$.

By comparing both schemes we can observe the following differences: The discrete density involved in the mixed variational problem for velocity pressure (1.13)-(1.14), which requires the property of the maximum principle, are truncated, whereas this truncation is not necessary for scheme (1.7)-(1.10). Moreover, in (1.12) the convective term for the density scheme is considered in the explicit form $\left(\boldsymbol{u}_{h}^{n} \cdot \nabla \rho_{h}^{n}, \bar{\rho}_{h}\right)$, and it is now taken in the semi-implicit form $\left(\boldsymbol{w}_{h}^{n} \cdot \nabla \rho_{h}^{n+1}, \bar{\rho}_{h}\right)$, where $\boldsymbol{w}_{h}^{n}$ is a projection of $\boldsymbol{u}_{h}^{n}$ onto a discrete zero-divergence space. This space is chosen to hold $\left(\nabla \cdot \boldsymbol{w}_{h}^{n}, \bar{\rho}_{h} \bar{\rho}_{h}\right)=0$ for all $\bar{\rho}_{h} \in W_{h}$.

Concerning the numerical analysis we remark on the following three main differences between both schemes:

1. The argument to obtain pointwise estimates for the discrete density under the constraints (S) done in subsection 3.4 of this paper is completely new. Moreover, the extension of this argument to the scheme studied in [11] is not clear even assuming some constraints on the discrete parameters. This justifies the presence of the truncation operator in the discrete momentum system (1.13). On the other hand, the scheme (1.12)-(1.14) of [11] is unconditionally stable, and now the scheme (1.7)-(1.10) is stable and convergent under the constraint (S).

2. Strong estimates for the discrete density are obtained in two different ways in [11] and in the present paper. In [11], we used the discrete version of the Gagliardo-Nirenberg interpolation inequality $\|\nabla \rho\|_{L^{4}}^{2} \leq C\|\rho\|_{H^{1}}\|\Delta \rho\|_{L^{2}}$ which does not need pointwise estimates for the discrete density. Since this interpolation is exclusive for two-dimensional domains, we cannot use it for three-dimensional domains. Accordingly, we change this Gagliardo-Nirenberg interpolation by a discrete version of the interpolation inequality $\|\nabla \rho\|_{L^{4}}^{2} \leq$ $C\|\rho\|_{L^{\infty}}\|\Delta \rho\|_{L^{2}}$ and make a discrete integration by parts (which mimics the argument of the exact problem to obtain strong estimates of the density). Observe that we have to assure a maximum principle or at least pointwise estimates for the discrete density in order for this other interpolation to work.

3. Another difference is the asymptotic behavior with respect to the diffusion parameter $\lambda$ (jointly with the discretization parameters $(k, h)$ ). Due to the fact that the convective term of the discrete density equation is handled in 
different ways, we find that the strong estimates of the discrete density furnished in [11] degenerate when $\lambda \rightarrow 0$, and we cannot pass to the limit towards a weak solution of the density-dependent Navier-Stokes problem (1.6). However, now the dependence of $\lambda$ is improved, and the scheme (1.14)-(1.10) gives a numerical approximation for the density-dependent Navier-Stokes problem (1.6) by means of continuous finite elements.

The rest of the paper can be described as follows. The main ideas for the mathematical analysis of problem (1.1)-(1.3) are provided in section 2. In section 3, by using appropriate auxiliary schemes, we establish conditional stability estimates, energy estimates for the velocity, and pointwise estimates for the density. In section 4, strong estimates for the density are obtained, by using the discrete Laplacian of the density. In sections 5, 6, and 7, weak and strong convergences and the passage to the limit are shown, respectively, concluding the proof of Theorem 1.1. In section 8 , we study the asymptotic behavior as the diffusion parameter $\lambda$ goes to zero, proving Theorem 1.2.

2. Analysis of the continuous model. To define the concept of a weak solution of problem (1.1)-(1.3), we introduce the following function spaces:

$$
\begin{aligned}
\boldsymbol{H} & =\left\{\boldsymbol{u}: \boldsymbol{u} \in \boldsymbol{L}^{2}(\Omega), \nabla \cdot \boldsymbol{u}=0 \text { in } \Omega, \boldsymbol{u} \cdot \boldsymbol{n}=0 \text { on } \Gamma\right\}, \\
\boldsymbol{V} & =\left\{\boldsymbol{u}: \boldsymbol{u} \in \boldsymbol{H}_{0}^{1}(\Omega), \nabla \cdot \boldsymbol{u}=0 \text { in } \Omega\right\}, \\
L_{0}^{2}(\Omega) & =\left\{p: p \in L^{2}(\Omega), \int_{\Omega} p(\boldsymbol{x}) d \boldsymbol{x}=0\right\}, \\
H_{N}^{2}(\Omega) & =\left\{\rho \in H^{2}(\Omega): \frac{\partial \rho}{\partial \boldsymbol{n}}=0 \text { on } \Gamma, \int_{\Omega} \rho(\boldsymbol{x}) d \boldsymbol{x}=\int_{\Omega} \rho_{0}(\boldsymbol{x}) d \boldsymbol{x}\right\} .
\end{aligned}
$$

In $\boldsymbol{V}$ the $\|\boldsymbol{u}\|_{H^{1}(\Omega)}$-norm is equivalent to $|\nabla \boldsymbol{u}|$ (which will be denoted by $\left.\|\boldsymbol{u}\|\right) . H_{N}^{2}(\Omega)$ is an affine space: $H_{N}^{2}(\Omega)=\frac{1}{|\Omega|} \int_{\Omega} \rho_{0}(\boldsymbol{x}) d \boldsymbol{x}+H_{N, 0}^{2}(\Omega)$, and in $H_{N, 0}^{2}(\Omega)$ (zero-average function space) the norm $\|\rho\|_{H^{1}(\Omega)}$ is equivalent to $|\nabla \rho|$ and the norm $\|\rho\|_{H^{2}(\Omega)}$ is equivalent to $|\Delta \rho|$. In particular, in $H_{N}^{2}(\Omega)$ the following norms are equivalents: $\left\|\rho-\frac{1}{|\Omega|} \int_{\Omega} \rho_{0}\right\|_{H^{1}(\Omega)} \sim|\nabla \rho|$ and $\|\nabla \rho\|_{H^{1}(\Omega)} \sim|\Delta \rho|$.

DEFINITION 2.1. A pair $(\rho, \boldsymbol{u})$ is called a weak solution of $(1.1)-(1.3)$ in $(0, T)$ if it verifies:

(a) $\boldsymbol{u} \in L^{\infty}(0, T ; \boldsymbol{H}) \cap L^{2}(0, T ; \boldsymbol{V}), \rho \in L^{\infty}\left(0, T ; H^{1}(\Omega)\right) \cap L^{2}\left(0, T ; H_{N}^{2}(\Omega)\right)$, with $0<m \leq \rho(\boldsymbol{x}, t) \leq M$, a.e. $(\boldsymbol{x}, t) \in Q$.

(b) $\forall \phi \in C^{1}([0, T] ; \boldsymbol{V})$ such that $\phi(T)=0$,

$$
\begin{aligned}
& \int_{0}^{T}\left\{-\left(\boldsymbol{u}, \rho \phi_{t}+(\rho \boldsymbol{u}-\lambda \nabla \rho) \cdot \nabla \phi\right)+\left(\mu \nabla \boldsymbol{u}-\lambda \rho(\nabla \boldsymbol{u})^{t}, \nabla \phi\right)\right\} d t \\
& =\int_{0}^{T}(\rho \boldsymbol{f}, \phi) d t+\left(\rho_{0} \boldsymbol{u}_{0}, \phi(0)\right) .
\end{aligned}
$$

(c) The equation of mass diffusion $(1.1)_{c}$ is verified a.e. in $Q$.

Remark 2.2. As usual, the pressure $p$ can be obtained by using (b) and De Rham's lemma [18].

We state the existence of (global in time) weak solutions of problem (1.1)-(1.3) (see $[12,1])$.

THEOREM 2.3. Let $\boldsymbol{u}_{0} \in \boldsymbol{H}, \rho_{0} \in H^{1}(\Omega)$ satisfying (1.4), and $\boldsymbol{f} \in L^{2}\left(0, T ; \boldsymbol{L}^{6 / 5}\right.$ $(\Omega))$. Suppose that the constants $\lambda, \mu, m$, and $M$ satisfy (1.5). Then there exists at least a weak solution of (1.1)-(1.3) in $(0, T)$. 
Proof (outline of proof). The proof is divided into four steps:

(a) Pointwise estimates for the density. From the maximum principle applied to the density equation $(1.1)_{c}$ and hypothesis (1.4), one gets

$$
0<m \leq \rho(\boldsymbol{x}, t) \leq M \quad \text { in } \quad Q .
$$

(b) Weak estimates for the velocity. Adding the momentum system $(1.1)_{a}$ by $\boldsymbol{u}$ to the density equation $(1.1)_{c}$ by $\frac{1}{2} \boldsymbol{u} \cdot \boldsymbol{u}$, one arrives at the following energy equality:

$$
\frac{1}{2} \frac{d}{d t} \int_{\Omega} \rho|\boldsymbol{u}|^{2} d \boldsymbol{x}+\mu\|\boldsymbol{u}\|^{2}=\lambda \int_{\Omega} \rho(\nabla \boldsymbol{u})^{t}: \nabla \boldsymbol{u} d \boldsymbol{x}+(\rho \boldsymbol{f}, \boldsymbol{u}) .
$$

The first term on the right-hand side of (2.1) can be rewritten as

$$
\lambda \int_{\Omega} \rho(\nabla \boldsymbol{u})^{t}: \nabla \boldsymbol{u} d \boldsymbol{x}=\lambda \int_{\Omega}\left(\rho-\frac{M+m}{2}\right)(\nabla \boldsymbol{u})^{t}: \nabla \boldsymbol{u} d \boldsymbol{x} \leq \lambda \frac{M-m}{2}\|u\|^{2},
$$

where we have used the pointwise inequality $|\rho-(M+m) / 2| \leq(M-m) / 2$ (obtained from $m \leq \rho \leq M)$. By imposing the constraint on the coefficients (1.5), one arrives at the estimate

$$
\max _{0 \leq t \leq T}|\boldsymbol{u}(t)|^{2}+\int_{0}^{T}\|\boldsymbol{u}(t)\|^{2} d t \leq C .
$$

(c) Strong estimates for the density. By multiplying the density equation (1.1) by $-\Delta \rho$ and bounding the convective term (previously integrated by parts) thanks to the interpolation inequality

$$
\|\nabla \rho\|_{L^{4}(\Omega)} \leq C\|\rho\|_{\infty}^{1 / 2}|\Delta \rho|^{1 / 2} \leq C|\Delta \rho|^{1 / 2},
$$

as

$$
\int_{\Omega} \boldsymbol{u} \cdot \nabla \rho \Delta \rho \leq C \int_{\Omega}|\nabla \boldsymbol{u}||\nabla \rho|^{2} \leq C|\nabla \boldsymbol{u}|\|\nabla \rho\|_{L^{4}}^{2} \leq C|\nabla \boldsymbol{u}||\Delta \rho|,
$$

the following estimate holds:

$$
\max _{0 \leq t \leq T}|\nabla \rho(t)|^{2}+\int_{0}^{T}|\Delta \rho(t)|^{2} d t \leq C_{\lambda} .
$$

(d) Compactness for the velocity. By using a rather technical argument [1], one can get the following estimate of the "time fractional derivative":

$$
\int_{0}^{T-\delta}|\boldsymbol{u}(t+\delta)-\boldsymbol{u}(t)|^{2} d t \leq C_{\lambda} \delta^{1 / 2} \quad \forall \delta \in(0, T),
$$

which implies [17] compactness for the velocity $\boldsymbol{u}$ in $L^{2}\left(0, T ; \boldsymbol{L}^{2}(\Omega)\right)$.

From here, it is rather standard to obtain the existence of weak solutions, by using, for instance, the semi-Galerkin method [1].

3. Weak and pointwise estimates. Since (1.7), (1.8), and (1.9)-(1.10) can be reduced to three independent algebraic linear systems, it suffices to check the uniqueness of the solution to guarantee that these problems are well-posed. In particular, the uniqueness will be a consequence of the weak and pointwise estimates that we will obtain in this section. 
3.1. Hypotheses. Throughout this work the following hypotheses will be assumed:

(H0) Hypotheses for the data: Assume (1.5), and let $\widetilde{M}>M$ and $0<\widetilde{m}<m$ such that $\lambda \frac{\widetilde{M}-\widetilde{m}}{2}<\mu$. Let $\boldsymbol{u}_{0} \in \boldsymbol{V}, \rho_{0} \in H^{1}(\Omega)$, with $0<m \leq \rho_{0} \leq M$ in $\Omega$, and $\boldsymbol{f} \in L^{2}\left(0, T ; \boldsymbol{L}^{6 / 5}(\Omega)\right)$.

(H1) Assume that $\Omega$ is an open, bounded set of $\mathbb{R}^{3}$ whose boundary is polyhedral and such that the continuous dependence in $H^{2}$-norm of the PoissonNeumann problem and in the $\boldsymbol{H}^{2} \times H^{1}$-norm of the Stokes problem holds (see (4.4) and (3.6), respectively). This is verified, for example, if $\Omega$ is convex [8].

(H2) The triangulation of $\Omega$ and the discrete spaces verify

- the inverse inequalities:

$$
\begin{aligned}
\left|\nabla \bar{\rho}_{h}\right| & \leq C h^{-1}\left|\bar{\rho}_{h}\right|, \quad\left\|\nabla \bar{\rho}_{h}\right\|_{L^{3}(\Omega)} \leq C h^{-1 / 2}\left|\nabla \bar{\rho}_{h}\right| \quad \forall \bar{\rho}_{h} \in W_{h}, \\
\left\|\bar{\rho}_{h}\right\|_{L^{\infty}(\Omega)} & \leq C h^{-1 / 2}\left\|\bar{\rho}_{h}\right\|_{H^{1}(\Omega)} \quad \forall \bar{\rho}_{h} \in W_{h},
\end{aligned}
$$

- and the interpolation errors:

$$
\begin{aligned}
\left\|\overline{\boldsymbol{u}}-\widetilde{J}_{h} \overline{\boldsymbol{u}}\right\|_{H^{1}(\Omega)}+\left\|\overline{\boldsymbol{u}}-J_{h} \overline{\boldsymbol{u}}\right\|_{H^{1}(\Omega)} & \leq C h|\overline{\boldsymbol{u}}|_{H^{2}(\Omega)} \quad \forall \overline{\boldsymbol{u}} \in \boldsymbol{H}^{2}(\Omega) \cap \boldsymbol{H}_{0}^{1}(\Omega), \\
\left|\bar{p}-\widetilde{K}_{h} \bar{p}\right|+\left|\bar{p}-K_{h} \bar{p}\right| & \leq C h|\bar{p}|_{H^{1}(\Omega)} \quad \forall \bar{p} \in H^{1}(\Omega) \cap L_{0}^{2}(\Omega), \\
\left\|\bar{\rho}-I_{h} \bar{\rho}\right\|_{L^{\infty}(\Omega) \cap W^{1,3}(\Omega)} & \leq C h^{1 / 2}|\bar{\rho}|_{H^{2}(\Omega)} \quad \forall \bar{\rho} \in H^{2}(\Omega), \\
\left|\bar{\rho}-I_{h} \bar{\rho}\right|+h\left\|\bar{\rho}-I_{h} \bar{\rho}\right\|_{H^{1}(\Omega)} & \leq C h^{2}|\bar{\rho}|_{H^{2}(\Omega)} \quad \forall \bar{\rho} \in H^{2}(\Omega),
\end{aligned}
$$

where $J_{h}, \widetilde{J}_{h}, K_{h}, \widetilde{K}_{h}$, and $I_{h}$ are interpolation operators from $\boldsymbol{H}^{2}(\Omega) \cap$ $\boldsymbol{H}_{0}^{1}(\Omega)$ into $\boldsymbol{V}_{h}, \boldsymbol{H}^{2}(\Omega) \cap \boldsymbol{H}_{0}^{1}(\Omega)$ into $\widetilde{\boldsymbol{V}}_{h}, H^{1}(\Omega) \cap L_{0}^{2}(\Omega)$ into $M_{h}$, $H^{1}(\Omega) \cap L_{0}^{2}(\Omega)$ into $\widetilde{M}_{h}$, and $H^{2}(\Omega)$ into $W_{h}$, respectively. Here and in what follows, we denote by $|v|_{H^{k}(\Omega)}=\sum_{|\alpha|=k}\left|D^{\alpha} v\right|$ the standard seminorm of higher order derivatives.

(H3) Inf-sup conditions. There exist $\beta>0$ and $\widetilde{\beta}>0$ (independent of $h$ ) such that, $\forall \bar{p}_{h} \in M_{h}$ and $\forall \bar{q}_{h} \in \widetilde{M}_{h}$,

$$
\left\|\bar{p}_{h}\right\|_{L_{0}^{2}(\Omega)} \leq \beta \sup _{\overline{\boldsymbol{u}}_{h} \in \boldsymbol{V}_{h} \backslash\{0\}} \frac{\left(\bar{p}_{h}, \nabla \cdot \overline{\boldsymbol{u}}_{h}\right)}{\left\|\overline{\boldsymbol{u}}_{h}\right\|}, \quad\left\|\bar{q}_{h}\right\|_{L_{0}^{2}(\Omega)} \leq \widetilde{\beta} \sup _{\overline{\boldsymbol{w}}_{h} \in \widetilde{\boldsymbol{V}}_{h \backslash\{0\}}} \frac{\left(\bar{q}_{h}, \nabla \cdot \overline{\boldsymbol{w}}_{h}\right)}{\left\|\overline{\boldsymbol{w}}_{h}\right\|} .
$$

(H4) Compatibility condition between $\widetilde{M}_{h}$ and $W_{h}:\left(W_{h} \cdot W_{h}\right) \cap L_{0}^{2}(\Omega) \subset \widetilde{M}_{h}$, i.e.,

$$
\forall \bar{\rho}_{h}^{1}, \bar{\rho}_{h}^{2} \in W_{h}, \quad \bar{\rho}_{h}^{1} \bar{\rho}_{h}^{2}-\frac{1}{|\Omega|} \int_{\Omega} \bar{\rho}_{h}^{1}(\boldsymbol{x}) \bar{\rho}_{h}^{2}(\boldsymbol{x}) d \boldsymbol{x} \in \widetilde{M}_{h} .
$$

(H5) Compatibility condition between $\left(M_{h}, \widetilde{M}_{h}\right)$ :

$$
M_{h} \subset \widetilde{M}_{h} .
$$

For instance, a way of defining the discrete spaces $\left(W_{h}, \boldsymbol{V}_{h}, M_{h}, \widetilde{\boldsymbol{V}}_{h}, \widetilde{M}_{h}\right)$ verifying (H2)-(H5) is the following: Let $\left\{\mathcal{T}_{h}\right\}_{h>0}$ be a regular, quasi-uniform family of triangulations of $\Omega$, with $h=\max _{K \in \mathcal{T}_{h}} h_{K}\left(h_{K}=\right.$ diameter of $\left.K\right)$, and

$$
X_{h}^{l}=\left\{x_{h} \in C^{0}(\bar{\Omega}) \text { such that }\left.x_{h}\right|_{K} \in \mathbb{P}_{l}(K) \forall K \in \mathcal{T}_{h},\right\} .
$$

Copyright (c) by SIAM. Unauthorized reproduction of this article is prohibited. 
Then we define $W_{h}=X_{h}^{1}$. There are several possibilities to define $\left(\boldsymbol{V}_{h}, M_{h}\right)$ [8], by using the Taylor-Hood element $\left(\mathbb{P}_{2} \times \mathbb{P}_{1}\right)$ or the minielement $\left(\mathbb{P}_{1}+\right.$ bubble $\left.\times \mathbb{P}_{1}\right)$, for instance. For the spaces $\left(\widetilde{\boldsymbol{V}}_{h}, \widetilde{M}_{h}\right)$ we choose $\widetilde{\boldsymbol{V}}_{h}=\boldsymbol{X}_{h}^{3} \cap \boldsymbol{H}_{0}^{1}(\Omega)$ and $\widetilde{M}_{h}=X_{h}^{2} \cap L_{0}^{2}(\Omega)$.

Note that if $\boldsymbol{V}_{h}=\widetilde{\boldsymbol{V}}_{h}$ and $M_{h}=\widetilde{M}_{h}$ are chosen, we need not consider the projection problem (1.7).

Remark 3.1. Hypothesis (H4) implies that $\left(\nabla \cdot \boldsymbol{w}_{h}^{n}, \bar{\rho}_{h}^{1} \bar{\rho}_{h}^{2}\right)=0$ for all $\bar{\rho}_{h}^{1}, \bar{\rho}_{h}^{2} \in W_{h}$ (this property will play an important role in our analysis). Indeed, we shall write

$$
\begin{aligned}
0 & =\left(\nabla \cdot \boldsymbol{w}_{h}^{n}, \bar{\rho}_{h}^{1} \bar{\rho}_{h}^{2}-\frac{1}{|\Omega|} \int_{\Omega} \bar{\rho}_{h}^{1} \bar{\rho}_{h}^{2}\right)=\left(\nabla \cdot \boldsymbol{w}_{h}^{n}, \bar{\rho}_{h}^{1} \bar{\rho}_{h}^{2}\right)-\frac{1}{|\Omega|} \int_{\Omega} \bar{\rho}_{h}^{1} \bar{\rho}_{h}^{2} \int_{\Omega} \nabla \cdot \boldsymbol{w}_{h}^{n} \\
& =\left(\nabla \cdot \boldsymbol{w}_{h}^{n}, \bar{\rho}_{h}^{1} \bar{\rho}_{h}^{2}\right) .
\end{aligned}
$$

As a consequence, by taking $\bar{\rho}_{h}=1$ in (1.8) we have that $\int_{\Omega} \rho_{h}^{n}=\int_{\Omega} \rho_{h}^{0}$, for each $n$. This property is the discrete version of the continuous one $\int_{\Omega} \rho\left(\boldsymbol{x}, t_{1}\right) d \boldsymbol{x}=$ $\int_{\Omega} \rho\left(\boldsymbol{x}, t_{2}\right) d \boldsymbol{x}$ for any $t_{1}, t_{2} \in[0, T]$, whose physical meaning is the conservation of mass.

3.2. Auxiliary truncated scheme. To prove a priori estimates for scheme (1.7)-(1.10), we will introduce an auxiliary scheme in which some of the densities appearing in the discrete problem of the momentum system are truncated between $\widetilde{m}$ and $\widetilde{M}$ as follows:

Initialization: Let $\boldsymbol{u}_{h}^{0}$ and $\rho_{h}^{0}$ be given as in scheme (1.7)-(1.10).

Time step $n+1$ : Given $\left(\rho_{h}^{n}, \boldsymbol{u}_{h}^{n}, p_{h}^{n}\right) \in W_{h} \times \boldsymbol{V}_{h} \times M_{h}$,

1. find $\left(\boldsymbol{w}_{h}^{n}, q_{h}^{n}\right) \in \widetilde{\boldsymbol{V}}_{h} \times \widetilde{M}_{h}$ such that, for each $\left(\overline{\boldsymbol{w}}_{h}, \bar{q}_{h}\right) \in \widetilde{\boldsymbol{V}}_{h} \times \widetilde{M}_{h}$,

$$
\left(\nabla \boldsymbol{w}_{h}^{n}, \nabla \overline{\boldsymbol{w}}_{h}\right)-\left(q_{h}^{n}, \nabla \cdot \overline{\boldsymbol{w}}_{h}\right)=\left(\nabla \boldsymbol{u}_{h}^{n}, \nabla \overline{\boldsymbol{w}}_{h}\right), \quad\left(\nabla \cdot \boldsymbol{w}_{h}^{n}, \bar{q}_{h}\right)=0
$$

2. find $\rho_{h}^{n+1} \in W_{h}$ such that, for each $\bar{\rho}_{h} \in W_{h}$,

$$
\left(\frac{\rho_{h}^{n+1}-\rho_{h}^{n}}{k}, \bar{\rho}_{h}\right)+\left(\boldsymbol{w}_{h}^{n} \cdot \nabla \rho_{h}^{n+1}, \bar{\rho}_{h}\right)+\lambda\left(\nabla \rho_{h}^{n+1}, \nabla \bar{\rho}_{h}\right)=0
$$

3. find $\left(\boldsymbol{u}_{h}^{n+1}, p_{h}^{n+1}\right) \in \boldsymbol{V}_{h} \times M_{h}$ such that, for each $\left(\overline{\boldsymbol{u}}_{h}, \bar{p}_{h}\right) \in \boldsymbol{V}_{h} \times M_{h}$,

$$
\left\{\begin{aligned}
&\left(\left[\rho_{h}^{n}\right]_{T} \frac{\boldsymbol{u}_{h}^{n+1}-\boldsymbol{u}_{h}^{n}}{k}, \overline{\boldsymbol{u}}_{h}\right)+\frac{1}{2}\left(\frac{\left[\rho_{h}^{n+1}\right]_{T}-\left[\rho_{h}^{n}\right]_{T}}{k} \boldsymbol{u}_{h}^{n+1}, \overline{\boldsymbol{u}}_{h}\right) \\
&+a\left(\left[\rho_{h}^{n+1}\right]_{T}, \boldsymbol{u}_{h}^{n+1}, \overline{\boldsymbol{u}}_{h}\right) \\
&+c\left(\rho_{h}^{n+1} \boldsymbol{u}_{h}^{n}-\lambda \nabla \rho_{h}^{n+1}, \boldsymbol{u}_{h}^{n+1}, \overline{\boldsymbol{u}}_{h}\right)=\left(\left[\rho_{h}^{n+1}\right]_{T} \boldsymbol{f}^{n+1}, \overline{\boldsymbol{u}}_{h}\right)+\left(p_{h}^{n+1}, \nabla \cdot \overline{\boldsymbol{u}}_{h}\right),
\end{aligned}\right.
$$

$$
\left(\nabla \cdot \boldsymbol{u}_{h}^{n+1}, q_{h}\right)=0
$$

Here the truncation $[\cdot]_{T}$ is defined as follows: Given $\rho_{h} \in W_{h}$, then

$$
\left[\rho_{h}\right]_{T}(\boldsymbol{x})=\left\{\begin{array}{lll}
\rho_{h}(\boldsymbol{x}) & \text { if } & \rho_{h}(\boldsymbol{x}) \in[\widetilde{m}, \widetilde{M}], \\
\widetilde{m} & \text { if } & \rho_{h}(\boldsymbol{x})<\widetilde{m}, \\
\widetilde{M} & \text { if } & \rho_{h}(\boldsymbol{x})>\widetilde{M} .
\end{array}\right.
$$

The idea was to truncate in those density terms which required to hold either $L^{\infty}$ estimates or positivity in order to obtain weak energy estimates (see the proof of Theorem 2.3). 
We may again deduce that (3.1), (3.2), and (3.3)-(3.4) are well-posed problems, obtaining a priori estimates.

3.3. Weak estimates for the truncated scheme.

LEMma 3.2. The solution of scheme (3.1)-(3.4) verifies the following estimates:
(i) $\max _{0 \leq n \leq N}\left|\boldsymbol{u}_{h}^{n}\right| \leq C$,
(ii) $k \sum_{n=0}^{N}\left\|\boldsymbol{u}_{h}^{n}\right\|^{2} \leq C$,
(iii) $\sum_{n=0}^{N-1}\left|\boldsymbol{u}_{h}^{n+1}-\boldsymbol{u}_{h}^{n}\right|^{2} \leq C$,
(iv) $\max _{0 \leq n \leq N}\left|\rho_{h}^{n}\right| \leq C$
(v) $\lambda k \sum_{n=0}^{N-1}\left|\nabla \rho_{h}^{n+1}\right|^{2} \leq C$,
(vi) $\sum_{n=0}^{N-1}\left|\rho_{h}^{n+1}-\rho_{h}^{n}\right|^{2} \leq C$,

where $C>0$ depends on the data $\left(\rho_{0}, \boldsymbol{u}_{0}, \boldsymbol{f}\right)$ but is independent of $k, h$, and $\lambda$.

Proof. To obtain a priori estimates for the velocity $\left(\boldsymbol{u}_{h}^{n}\right)$, we take $\overline{\boldsymbol{u}}_{h}=2 k \boldsymbol{u}_{h}^{n+1}$ and $\bar{p}_{h}=p_{h}^{n+1}$ as test functions in (3.3)-(3.4), resulting in [11]:

$$
\begin{aligned}
& \left|\sqrt{\left[\rho_{h}^{n+1}\right]_{T}} \boldsymbol{u}_{h}^{n+1}\right|^{2}-\left|\sqrt{\left[\rho_{h}^{n}\right]_{T}} \boldsymbol{u}_{h}^{n}\right|^{2}+\left|\sqrt{\left[\rho_{h}^{n}\right]_{T}}\left(\boldsymbol{u}_{h}^{n+1}-\boldsymbol{u}_{h}^{n}\right)\right|^{2}+\mu_{1} k\left\|\boldsymbol{u}_{h}^{n+1}\right\|^{2} \\
& \leq 2 k\left(\left[\rho_{h}^{n+1}\right]_{T} \boldsymbol{f}^{n+1}, \boldsymbol{u}_{h}^{n+1}\right) \leq 2 k\left\|\left[\rho_{h}^{n+1}\right]_{T}\right\|_{L^{\infty}(\Omega)}\left\|\boldsymbol{f}^{n+1}\right\|_{L^{6 / 5}(\Omega)}\left\|\boldsymbol{u}_{h}^{n+1}\right\|_{L^{6}(\Omega)} \\
& \leq \frac{\mu_{1} k}{2}\left\|\boldsymbol{u}_{h}^{n+1}\right\|^{2}+C k\left\|\boldsymbol{f}^{n+1}\right\|_{L^{6 / 5}(\Omega)}^{2} .
\end{aligned}
$$

Consequently,

$$
\begin{aligned}
& \left|\sqrt{\left[\rho_{h}^{n+1}\right]_{T}} \boldsymbol{u}_{h}^{n+1}\right|^{2}-\left|\sqrt{\left[\rho_{h}^{n}\right]_{T}} \boldsymbol{u}_{h}^{n}\right|^{2}+\left|\sqrt{\left[\rho_{h}^{n}\right]_{T}}\left(\boldsymbol{u}_{h}^{n+1}-\boldsymbol{u}_{h}^{n}\right)\right|^{2} \\
& +\frac{\mu_{1}}{2} k\left\|\boldsymbol{u}_{h}^{n+1}\right\|^{2} \leq C k\left\|\boldsymbol{f}^{n+1}\right\|_{L^{6 / 5}(\Omega)}^{2} .
\end{aligned}
$$

By adding (3.5) for $n=0, \ldots, r$ with any $r<N$, estimates (i), (ii), and (iii) hold.

On the other hand, to obtain weak energy estimates for the density $\left(\rho_{h}^{n}\right)$, we take $\bar{\rho}_{h}=2 k \rho_{h}^{n+1}$ in $(3.2)$ and use the fact that $\left(\nabla \cdot \boldsymbol{w}_{h}^{n},\left(\rho_{h}^{n+1}\right)^{2}\right)=0$ (see Remark 3.1):

$$
\left|\rho_{h}^{n+1}\right|^{2}-\left|\rho_{h}^{n}\right|^{2}+\left|\rho_{h}^{n+1}-\rho_{h}^{n}\right|^{2}+2 \lambda k\left|\nabla \rho_{h}^{n+1}\right|^{2}=0 .
$$

By adding over $n$, one deduces estimates (iv), (v), and (vi).

COROLlary 3.3. The following estimates hold:

$$
\text { (vii) } \max _{0 \leq n \leq N}\left|\boldsymbol{w}_{h}^{n}\right| \leq C, \quad \text { (viii) } k \sum_{n=0}^{N}\left\|\boldsymbol{w}_{h}^{n}\right\|^{2} \leq C,
$$

where $C>0$ is independent of $k, h$, and $\lambda$.

Proof. By taking $\overline{\boldsymbol{w}}_{h}=\boldsymbol{w}_{h}^{n}$ in (1.7) and using (H5), $\left(\nabla\left(\boldsymbol{w}_{h}^{n}-\boldsymbol{u}_{h}^{n}\right), \nabla \boldsymbol{w}_{h}^{n}\right)=0$, and hence one has $\left|\nabla \boldsymbol{w}_{h}^{n}\right| \leq\left|\nabla \boldsymbol{u}_{h}^{n}\right|$. So from (ii) we get (viii). Now we are going to get estimate (vii) by using a duality technique and the constraint (S). Indeed, let $(\boldsymbol{z}, \xi) \in\left(\boldsymbol{V} \cap \boldsymbol{H}^{2}(\Omega)\right) \times\left(L_{0}^{2}(\Omega) \cap H^{1}(\Omega)\right)$ be the strong solution of the Stokes problem

$$
-\Delta \boldsymbol{z}+\nabla \xi=\boldsymbol{w}_{h}^{n}-\boldsymbol{u}_{h}^{n}, \quad \nabla \cdot \boldsymbol{z}=0 \text { in } \Omega, \quad \boldsymbol{z}=0 \text { on } \Gamma .
$$

By taking $\boldsymbol{w}_{h}^{n}-\boldsymbol{u}_{h}^{n}$ as a test function in the variational formulation of (3.6), we get

$$
\left|\boldsymbol{w}_{h}^{n}-\boldsymbol{u}_{h}^{n}\right|^{2}=\left(\nabla \boldsymbol{z}, \nabla\left(\boldsymbol{w}_{h}^{n}-\boldsymbol{u}_{h}^{n}\right)\right)+\left(\xi, \nabla \cdot\left(\boldsymbol{w}_{h}^{n}-\boldsymbol{u}_{h}^{n}\right)\right) .
$$

Copyright $@$ by SIAM. Unauthorized reproduction of this article is prohibited. 
Let $\left(z_{h}, \xi_{h}\right) \in \widetilde{V_{h}} \times \widetilde{M}_{h}$ be the discrete approximation of (3.6) defined as

$$
\left\{\begin{aligned}
\left(\nabla \boldsymbol{z}_{h}, \nabla \overline{\boldsymbol{w}}_{h}\right)-\left(\xi_{h}, \nabla \cdot \overline{\boldsymbol{w}}_{h}\right) & =\left(\boldsymbol{w}_{h}^{n}-\boldsymbol{u}_{h}^{n}, \overline{\boldsymbol{w}}_{h}\right) \quad \forall \overline{\boldsymbol{w}}_{h} \in \widetilde{\boldsymbol{V}}_{h}, \\
\left(\nabla \cdot \boldsymbol{z}_{h}, \bar{q}_{h}\right) & =0 \quad \forall \bar{q}_{h} \in \widetilde{M}_{h} .
\end{aligned}\right.
$$

In view of hypothesis $(\mathrm{H} 5),\left(K_{h} \xi, \nabla \cdot\left(\boldsymbol{w}_{h}^{n}-\boldsymbol{u}_{h}^{n}\right)\right)=0$, and hence we write (3.7) as follows:

$\left|\boldsymbol{w}_{h}^{n}-\boldsymbol{u}_{h}^{n}\right|^{2}=\left(\nabla \boldsymbol{z}-\nabla \boldsymbol{z}_{h}, \nabla\left(\boldsymbol{w}_{h}^{n}-\boldsymbol{u}_{h}^{n}\right)\right)+\left(\nabla \boldsymbol{z}_{h}, \nabla\left(\boldsymbol{w}_{h}^{n}-\boldsymbol{u}_{h}^{n}\right)\right)+\left(\xi-K_{h} \xi, \nabla \cdot\left(\boldsymbol{w}_{h}^{n}-\boldsymbol{u}_{h}^{n}\right)\right)$,

where $K_{h}$ is the interpolation operator defined in hypothesis (H2). From (1.7), it follows that $\left(\nabla \boldsymbol{z}_{h}, \nabla\left(\boldsymbol{w}_{h}^{n}-\boldsymbol{u}_{h}^{n}\right)\right)=0$. Thus, we find

$$
\begin{aligned}
& \left|\boldsymbol{w}_{h}^{n}-\boldsymbol{u}_{h}^{n}\right|^{2} \leq\left|\nabla \boldsymbol{z}-\nabla \boldsymbol{z}_{h}\right|\left|\nabla\left(\boldsymbol{w}_{h}^{n}-\boldsymbol{u}_{h}^{n}\right)\right|+\left|\xi-K_{h} \xi\right|\left|\nabla \cdot\left(\boldsymbol{w}_{h}^{n}-\boldsymbol{u}_{h}^{n}\right)\right| \\
& \leq C h\left(\|\boldsymbol{z}\|_{H^{2}(\Omega)}+\|\xi\|_{H^{1}(\Omega)}\right)\left|\nabla\left(\boldsymbol{w}_{h}^{n}-\boldsymbol{u}_{h}^{n}\right)\right| \leq C h\left|\boldsymbol{w}_{h}^{n}-\boldsymbol{u}_{h}^{n}\right|\left|\nabla\left(\boldsymbol{w}_{h}^{n}-\boldsymbol{u}_{h}^{n}\right)\right|,
\end{aligned}
$$

where in the second line we have used the approximation property (see $[8]) \mid \nabla \boldsymbol{z}-$ $\nabla z_{h} \mid \leq C h\left(\|z\|_{H^{2}}+\|\xi\|_{H^{1}}\right)$, the interpolation error $\left|\xi-K_{h} \xi\right| \leq C h\|\xi\|_{H^{1}(\Omega)}$ assumed in (H2), and the $H^{2} \times H^{1}$ continuous dependency of the Stokes problem (3.6) $\|\boldsymbol{z}\|_{H^{2}(\Omega)}+\|\xi\|_{H^{1}(\Omega)} \leq C\left|\boldsymbol{w}_{h}^{n}-\boldsymbol{u}_{h}^{n}\right|$ assumed in (H1).

Therefore, we have

$$
\left|\boldsymbol{w}_{h}^{n}-\boldsymbol{u}_{h}^{n}\right| \leq C h\left|\nabla\left(\boldsymbol{w}_{h}^{n}-\boldsymbol{u}_{h}^{n}\right)\right| .
$$

Now, in view of (S), we may get $h \leq C k$ for $(h, k)$ small enough. Then, since $k^{1 / 2}\left|\nabla \boldsymbol{w}_{h}^{n}\right| \leq k^{1 / 2}\left|\nabla \boldsymbol{u}_{h}^{n}\right| \leq C$ (thanks to the estimate $\left|\nabla \boldsymbol{w}_{h}^{n}\right| \leq\left|\nabla \boldsymbol{u}_{h}^{n}\right|$ and estimate (ii) of Lemma 3.2), it is easy to see that $\left|\boldsymbol{w}_{h}^{n}\right| \leq C k^{1 / 2}+\left|\boldsymbol{u}_{h}^{n}\right| \leq C$, and we get estimate (vii).

3.4. Discrete maximum principle (of the truncated scheme). In this subsection we prove that the discrete density of scheme (3.2) has pointwise estimates by excess and defect with respect to the upper and lower bounds of the initial density $\rho_{0}$, respectively. Namely, we will see that $\widetilde{m} \leq \rho_{h}^{n} \leq \widetilde{M}$ in $\Omega$ for all $k$ and $h$ small enough satisfying constraint (S).

3.4.1. Study of an auxiliary time discrete scheme. We define a sequence $\left(\rho^{n}\right)$ associated to $\left(\boldsymbol{w}_{h}^{n}\right)$ by means of the following time discrete scheme:

Initialization: Let $\rho^{0}=\rho_{0}$.

Time step $n+1$ : Given $\rho^{n}$, we compute $\rho^{n+1} \in H^{2}(\Omega)$, verifying

$$
\frac{\rho^{n+1}-\rho^{n}}{k}+\boldsymbol{w}_{h}^{n} \cdot \nabla \rho^{n+1}-\lambda \Delta \rho^{n+1}=0 \text { in } \Omega, \quad \frac{\partial \rho^{n+1}}{\partial \boldsymbol{n}}=0 \text { on } \Gamma .
$$

Lemma 3.4. Let $\left\{\boldsymbol{w}_{h}^{n}\right\}_{n=0}^{N} \subset \boldsymbol{H}_{0}^{1}(\Omega)$ such that $k \sum_{n=0}^{N}\left\|\boldsymbol{w}_{h}^{n}\right\|^{2} \leq C$. Then there exists a unique solution $\rho^{n+1} \in H^{2}(\Omega)$ of (3.9), which also verifies:

$$
\begin{gathered}
0<m \leq \rho^{n+1}(\boldsymbol{x}) \leq M \quad \forall \boldsymbol{x} \in \Omega, \quad \forall n=0, \ldots, N-1, \\
\lambda^{2} k \sum_{n=0}^{N-1}\left\|\rho^{n+1}\right\|_{H^{2}(\Omega)}^{2} \leq C,
\end{gathered}
$$

where $C>0$ is a constant independent of $k, h$, and $\lambda$.

Proof. The proof of this lemma can be found in Appendix A. 
3.4.2. Error estimates between $\rho^{n+1}$ and $\boldsymbol{\rho}_{\boldsymbol{h}}^{n+1}$. Denote by $e_{\rho}^{n+1}=\rho^{n+1}-$ $\rho_{h}^{n+1}$ the difference between the solutions of problems (3.9) and (3.2). Our intention now is to state the following error estimate:

$$
\left\|e_{\rho}^{n+1}\right\|_{H^{1}(\Omega)} \leq C\left(\frac{h^{2}}{\lambda \sqrt{\lambda} k}+\frac{h \sqrt{h}}{\lambda^{2} k}+\frac{h h^{1 / 4}}{\lambda^{3 / 2} k^{3 / 4}}+\frac{h}{\lambda \sqrt{k}}\right) .
$$

Indeed, by subtracting (3.9) multiplied by $\bar{\rho}_{h} \in W_{h}$ and (3.2), one has

$$
\left(\frac{e_{\rho}^{n+1}-e_{\rho}^{n}}{k}, \bar{\rho}_{h}\right)+\left(\boldsymbol{w}_{h}^{n} \cdot \nabla e_{\rho}^{n+1}, \bar{\rho}_{h}\right)+\lambda\left(\nabla e_{\rho}^{n+1}, \nabla \bar{\rho}_{h}\right)=0
$$

for each $\bar{\rho}_{h} \in W_{h}$. By decomposing the convective term as

$$
\left(\boldsymbol{w}_{h}^{n} \cdot \nabla e_{\rho}^{n+1}, \bar{\rho}_{h}\right)=\left(\boldsymbol{w}_{h}^{n} \cdot \nabla\left(\rho^{n+1}-I_{h} \rho^{n+1}\right), \bar{\rho}_{h}\right)+\left(\boldsymbol{w}_{h}^{n} \cdot \nabla\left(I_{h} \rho^{n+1}-\rho_{h}^{n+1}\right), \bar{\rho}_{h}\right),
$$

taking $\bar{\rho}_{h}=2 k\left(e_{\rho}^{n+1}-\rho^{n+1}+I_{h} \rho^{n+1}\right)=2 k\left(I_{h} \rho^{n+1}-\rho_{h}^{n+1}\right)$, with $I_{h} \rho^{n+1} \in W_{h}$, and using the fact that $\left(\nabla \cdot \boldsymbol{w}_{h}^{n}, \bar{\rho}_{h}^{2}\right)=0$ for all $\bar{\rho}_{h} \in W_{h}$ (see Remark 3.1), we get

$$
\begin{aligned}
& \left|e_{\rho}^{n+1}\right|^{2}-\left|e_{\rho}^{n}\right|^{2}+\left|e_{\rho}^{n+1}-e_{\rho}^{n}\right|^{2}+2 \lambda k\left|\nabla e_{\rho}^{n+1}\right|^{2} \\
& \leq 2\left(e_{\rho}^{n+1}-e_{\rho}^{n}, \rho^{n+1}-I_{h} \rho^{n+1}\right)-2 k\left(\boldsymbol{w}_{h}^{n} \cdot \nabla\left(\rho^{n+1}-I_{h} \rho^{n+1}\right), e_{\rho}^{n+1}\right) \\
& -k\left(\nabla \cdot \boldsymbol{w}_{h}^{n},\left(\rho^{n+1}-I_{h} \rho^{n+1}\right)^{2}\right)+2 \lambda k\left(\nabla e_{\rho}^{n+1}, \nabla\left(\rho^{n+1}-I_{h} \rho^{n+1}\right)\right) .
\end{aligned}
$$

Next, by integrating by parts the second term on the right-hand side and bounding adequately, we infer that

$$
\begin{aligned}
& \left|e_{\rho}^{n+1}\right|^{2}-\left|e_{\rho}^{n}\right|^{2}+\left|e_{\rho}^{n+1}-e_{\rho}^{n}\right|^{2}+2 \lambda k\left|\nabla e_{\rho}^{n+1}\right|^{2} \\
& \leq 2\left|e_{\rho}^{n+1}-e_{\rho}^{n}\right|\left|\rho^{n+1}-I_{h} \rho^{n+1}\right|+C k\left\|\boldsymbol{w}_{h}^{n}\right\|\left\|e_{\rho}^{n+1}\right\|_{H^{1}(\Omega)}\left\|\rho^{n+1}-I_{h} \rho^{n+1}\right\|_{L^{3}(\Omega)} \\
& +k\left\|\boldsymbol{w}_{h}^{n}\right\|\left\|\rho^{n+1}-I_{h} \rho^{n+1}\right\|_{L^{4}(\Omega)}^{2}+2 \lambda k\left|\nabla e_{\rho}^{n+1}\right|\left|\nabla\left(\rho^{n+1}-I_{h} \rho^{n+1}\right)\right| \\
& \leq \frac{1}{2}\left|e_{\rho}^{n+1}-e_{\rho}^{n}\right|^{2}+C\left|\rho^{n+1}-I_{h} \rho^{n+1}\right|^{2}+C \lambda k\left|\nabla\left(\rho^{n+1}-I_{h} \rho^{n+1}\right)\right|^{2} \\
& +\frac{C}{\lambda} k\left\|\boldsymbol{w}_{h}^{n}\right\|^{2}\left\|\rho^{n+1}-I_{h} \rho^{n+1}\right\|_{L^{3}}^{2}+k\left\|\boldsymbol{w}_{h}^{n}\right\|\left\|\rho^{n+1}-I_{h} \rho^{n+1}\right\|_{L^{4}}^{2}+\lambda k\left|e_{\rho}^{n+1}\right|^{2}+\lambda k\left|\nabla e_{\rho}^{n+1}\right|^{2},
\end{aligned}
$$

and then, by taking into account the interpolation errors

$$
\left|\rho^{n+1}-I_{h} \rho^{n+1}\right|+h\left|\nabla\left(\rho^{n+1}-I_{h} \rho^{n+1}\right)\right| \leq C h^{2}\left\|\rho^{n+1}\right\|_{H^{2}},
$$

and

$$
\left\|\rho^{n+1}-I_{h} \rho^{n+1}\right\|_{L^{3}} \leq C h^{3 / 2}\left\|\rho^{n+1}\right\|_{H^{2}}, \quad\left\|\rho^{n+1}-I_{h} \rho^{n+1}\right\|_{L^{4}} \leq C h^{5 / 4}\left\|\rho^{n+1}\right\|_{H^{2}}
$$

(the last two are a consequence of the previous one and the 3D interpolation inequalities $\|\rho\|_{L^{3}} \leq C|\rho|^{1 / 2}\|\rho\|_{H^{1}}^{1 / 2}$ and $\left.\|\rho\|_{L^{4}} \leq C|\rho|^{1 / 4}\|\rho\|_{H^{1}}^{3 / 4}\right)$ and the estimate $k\left\|\boldsymbol{w}_{h}^{n}\right\|^{2} \leq C$ thanks to estimate (vii) of Corollary 3.3 , one arrives at

$$
\begin{aligned}
& \left|e_{\rho}^{n+1}\right|^{2}-\left|e_{\rho}^{n}\right|^{2}+\lambda k\left|\nabla e_{\rho}^{n+1}\right|^{2} \\
& \quad \leq C\left(h^{4}+\frac{h^{3}}{\lambda}+k^{1 / 2} h^{5 / 2}+\lambda k h^{2}\right)\left\|\rho^{n+1}\right\|_{H^{2}}^{2}+\lambda k\left|e_{\rho}^{n+1}\right|^{2} .
\end{aligned}
$$

Copyright $@$ ㅇ by SIAM. Unauthorized reproduction of this article is prohibited. 
By adding up for $n=0, \ldots, l$ and using the fact that $\lambda^{2} k \sum_{n=0}^{l}\left\|\rho^{n+1}\right\|_{H^{2}}^{2} \leq C$ and by virtue of the generalized discrete Gronwall lemma, we infer that for all $(k, \lambda)$, with $\lambda k<1$ (for instance, $\lambda k \leq 1 / 2$ ), there exists $C>0$ independent of $\lambda$ such that

$$
\left|e_{\rho}^{l+1}\right|^{2}+\lambda k \sum_{n=0}^{l}\left|\nabla e_{\rho}^{n+1}\right|^{2} \leq C\left(\frac{h^{4}}{\lambda^{2} k}+\frac{h^{3}}{\lambda^{3} k}+\frac{h^{5 / 2}}{\lambda^{2} k^{1 / 2}}+\frac{h^{2}}{\lambda}+\left|e_{\rho}^{0}\right|^{2}\right) .
$$

By taking $\left|e_{\rho}^{0}\right|^{2}=\left|\rho^{0}-\rho_{h}^{0}\right|^{2} \leq C h^{2}$, we deduce the bound

$$
\left|e_{\rho}^{n+1}\right|^{2}+\lambda k \sum_{n=0}^{l}\left|\nabla e_{\rho}^{n+1}\right|^{2} \leq C\left(\frac{h^{4}}{\lambda^{2} k}+\frac{h^{3}}{\lambda^{3} k}+\frac{h^{5 / 2}}{\lambda^{2} k^{1 / 2}}+\frac{h^{2}}{\lambda}\right),
$$

whence, in particular, (3.11) holds.

3.4.3. Pointwise estimates of the truncated scheme. Here we will prove the following pointwise estimates [6]:

$$
\begin{aligned}
& \rho_{h}^{n+1} \geq m-C h^{-1 / 2}\left(\frac{h^{2}}{\lambda^{3 / 2} k}+\frac{h \sqrt{h}}{\lambda^{2} k}+\frac{h h^{1 / 4}}{\lambda^{2} k^{3 / 4}}+\frac{h}{\lambda \sqrt{k}}\right), \\
& \rho_{h}^{n+1} \leq M+C h^{-1 / 2}\left(\frac{h^{2}}{\lambda^{3 / 2} k}+\frac{h \sqrt{h}}{\lambda^{2} k}+\frac{h h^{1 / 4}}{\lambda^{2} k^{3 / 4}}+\frac{h}{\lambda \sqrt{k}}\right) .
\end{aligned}
$$

To prove (3.12) it suffices to prove that

$$
\left\|\rho_{h}^{n+1}-\rho^{n+1}\right\|_{L^{\infty}(\Omega)} \leq C h^{-1 / 2}\left(\frac{h^{2}}{\lambda^{3 / 2} k}+\frac{h \sqrt{h}}{\lambda^{2} k}+\frac{h h^{1 / 4}}{\lambda^{2} k^{3 / 4}}+\frac{h}{\lambda \sqrt{k}}\right) .
$$

For this, from the triangle inequality

$$
\begin{aligned}
\left\|\rho_{h}^{n+1}-\rho^{n+1}\right\|_{L^{\infty}(\Omega)} & \leq\left\|\rho_{h}^{n+1}-I_{h} \rho^{n+1}\right\|_{L^{\infty}(\Omega)}+\left\|I_{h} \rho^{n+1}-\rho^{n+1}\right\|_{L^{\infty}(\Omega)} \\
& \leq\left\|\rho_{h}^{n+1}-I_{h} \rho^{n+1}\right\|_{L^{\infty}(\Omega)}+C \frac{h^{1 / 2}}{\lambda k^{1 / 2}},
\end{aligned}
$$

where in the last line we have used the approximation inequality $\left\|\rho^{n+1}-I_{h} \rho^{n+1}\right\|_{L^{\infty}} \leq$ $C h^{1 / 2}\left\|\rho^{n+1}\right\|_{H^{2}}$ and the estimate $\left\|\rho^{n+1}\right\|_{H^{2}} \leq \frac{C}{\lambda k^{1 / 2}}$ (see (3.10)). Hence, it suffices to obtain the inequality

$$
\left\|\rho_{h}^{n+1}-I_{h} \rho^{n+1}\right\|_{H^{1}(\Omega)} \leq C\left(\frac{h^{2}}{\lambda^{3 / 2} k}+\frac{h \sqrt{h}}{\lambda^{2} k}+\frac{h h^{1 / 4}}{\lambda^{2} k^{3 / 4}}+\frac{h}{\lambda \sqrt{k}}\right)
$$

and to use the inverse inequality (see [3]) $\left\|\bar{\rho}_{h}\right\|_{L^{\infty}(\Omega)} \leq C h^{-1 / 2}\left\|\bar{\rho}_{h}\right\|_{H^{1}(\Omega)} \forall \bar{\rho}_{h} \in W_{h}$. Let us prove (3.13). From the triangle inequality,

$$
\left\|\rho_{h}^{n+1}-I_{h} \rho^{n+1}\right\|_{H^{1}(\Omega)} \leq\left\|\rho_{h}^{n+1}-\rho^{n+1}\right\|_{H^{1}(\Omega)}+\left\|\rho^{n+1}-I_{h} \rho^{n+1}\right\|_{H^{1}(\Omega)},
$$

and, by using the error estimate (3.11) and the interpolation error $\left\|w-I_{h} w\right\|_{H^{1}(\Omega)} \leq$ $h\|w\|_{H^{2}(\Omega)}$ for $w=\rho^{n+1}$ jointly with the estimate $h\left\|\rho^{n+1}\right\|_{H^{2}} \leq C h / \lambda \sqrt{k}$ (thanks to (3.10)), one easily deduces (3.13).

Copyright $@$ by SIAM. Unauthorized reproduction of this article is prohibited. 
Now, by taking into account hypothesis (S), one has

$$
\lim _{(h, k) \rightarrow 0}\left(\frac{h^{2}}{k}+\frac{h \sqrt{h}}{k}+\frac{h h^{1 / 4}}{k^{3 / 4}}\right) /\left(\frac{h}{\sqrt{k}}\right)=0,
$$

and consequently, for each $(h, k)$ small enough,

$$
C h^{-1 / 2}\left(\frac{h^{2}}{\lambda^{3 / 2} k}+\frac{h \sqrt{h}}{\lambda^{2} k}+\frac{h}{\lambda \sqrt{k}}\right) \leq C h^{-1 / 2} \frac{h}{\sqrt{k}}=C \sqrt{\frac{h}{k}} .
$$

In particular, thanks to (3.12), by imposing $h \leq h_{0}, k \leq k_{0}$ such that

$$
C \sqrt{\frac{h}{k}} \leq \min \{m-\widetilde{m}, \widetilde{M}-M\},
$$

one gets $0<\widetilde{m} \leq \rho_{h}^{n+1} \leq \widetilde{M}$.

3.4.4. Identification between the truncated and nontruncated schemes. Now it is clear that if $\rho_{h}^{n+1}$ is the solution of the truncated scheme, then $\left[\rho_{h}^{n+1}\right]_{T}=$ $\rho_{h}^{n+1}$, and consequently the truncated scheme and the nontruncated scheme coincide, arriving at the following result.

THEOREM 3.5. Assume that $h \leq h_{0}, k \leq k_{0}$ satisfying (3.14) and $\lambda k \leq 1 / 2$; then scheme (1.7)-(1.10) is well-posed and verifies estimates (i)-(vi) of Lemma 3.2, (vii)-(viii) of Corollary 3.3, and $0<\widetilde{m} \leq \rho_{h}^{n+1} \leq \widetilde{M}$ in $\Omega$.

4. Strong estimates for the density. Let $-\Delta_{h}: W_{h} \rightarrow W_{h}$ be the linear operator defined as follows:

$$
-\left(\Delta_{h} \rho_{h}, \bar{\rho}_{h}\right)=\left(\nabla \rho_{h}, \nabla \bar{\rho}_{h}\right) \quad \forall \bar{\rho}_{h} \in W_{h} .
$$

Then the discrete density equation (1.8) can be rewritten as

$$
\left(\frac{\rho_{h}^{n+1}-\rho_{h}^{n}}{k}, \bar{\rho}_{h}\right)+\left(\boldsymbol{w}_{h}^{n} \cdot \nabla \rho_{h}^{n+1}, \bar{\rho}_{h}\right)-\lambda\left(\Delta_{h} \rho_{h}^{n+1}, \bar{\rho}_{h}\right)=0 .
$$

THEOREM 4.1. Under the hypotheses of Theorem 3.5, the solution $\rho_{h}^{n+1}$ of scheme (1.8) verifies the following estimates, for each $h$ and $k$ small enough:

$$
\begin{gathered}
\text { (ix) } \lambda \max _{0 \leq n \leq N}\left|\nabla \rho_{h}^{n}\right|^{2} \leq C, \quad\left(\text { x) } \lambda^{2} k \sum_{n=0}^{N}\left|\Delta_{h} \rho_{h}^{n+1}\right|^{2} \leq C,\right. \\
\text { (xi) } \lambda \sum_{n=0}^{N-1}\left|\nabla\left(\rho_{h}^{n+1}-\rho_{h}^{n}\right)\right|^{2} \leq C,
\end{gathered}
$$

where $C>0$ is independent of $h, k$, and $\lambda$.

Proof. By taking $\bar{\rho}_{h}=-2 k \Delta_{h} \rho_{h}^{n+1}$ in (4.2), we arrive at:

$\left|\nabla \rho_{h}^{n+1}\right|^{2}-\left|\nabla \rho_{h}^{n}\right|^{2}+\left|\nabla\left(\rho_{h}^{n+1}-\rho_{h}^{n}\right)\right|^{2}+2 \lambda k\left|\Delta_{h} \rho_{h}^{n+1}\right|^{2}=2 k\left(\boldsymbol{w}_{h}^{n} \cdot \nabla \rho_{h}^{n+1}, \Delta_{h} \rho_{h}^{n+1}\right):=I$.

To bound $I$, we use an idea given in [11], where a regular function associated to the discrete Laplacian function $-\Delta_{h} \rho_{h}^{n+1}$ is considered. But here, the type of estimates 
used in [11] must be changed, making use of the pointwise estimates of $\rho_{h}^{n+1}$. We define $\rho(h) \in H^{2}(\Omega)$ as the solution of the problem:

$$
-\Delta \rho(h)=-\Delta_{h} \rho_{h}^{n+1} \text { in } \Omega,\left.\quad \frac{\partial \rho(h)}{\partial \boldsymbol{n}}\right|_{\partial \Omega}=0, \quad \int_{\Omega} \rho(h)=\int_{\Omega} \rho_{h}^{0} .
$$

From the $H^{2}$-regularity of the previous problem $\left\|\rho(h)-\frac{1}{|\Omega|} \int \rho_{h}^{0}\right\|_{H^{2}(\Omega)} \leq C\left|\Delta_{h} \rho_{h}^{n+1}\right|$, and hence one has in particular

$$
|\rho(h)|_{H^{2}(\Omega)} \leq C\left|\Delta_{h} \rho_{h}^{n+1}\right| .
$$

We write $I$ as $I=2 k\left(\boldsymbol{w}_{h}^{n} \cdot \nabla \rho(h), \Delta \rho(h)\right)+2 k\left(\boldsymbol{w}_{h}^{n} \cdot \nabla\left(\rho_{h}^{n+1}-\rho(h)\right), \Delta_{h} \rho_{h}^{n+1}\right)$. By integrating by parts the first term on the right-hand side, and using (2.2),

$$
\begin{aligned}
2 k\left(\boldsymbol{w}_{h}^{n} \cdot \nabla \rho(h), \Delta \rho(h)\right) & =-2 k\left(\nabla \boldsymbol{w}_{h}^{n}, \nabla \rho(h) \otimes \nabla \rho(h)\right)-2 k\left(\left(\boldsymbol{w}_{h}^{n} \cdot \nabla\right) \nabla \rho(h), \nabla \rho(h)\right) \\
& =-2 k\left(\nabla \boldsymbol{w}_{h}^{n}, \nabla \rho(h) \otimes \nabla \rho(h)\right)+k\left(\nabla \cdot \boldsymbol{w}_{h}^{n},|\nabla \rho(h)|^{2}\right) \\
& \leq C k\left\|\boldsymbol{w}_{h}^{n}\right\|\|\nabla \rho(h)\|_{L^{4}(\Omega)}^{2} \leq C k\left\|\boldsymbol{w}_{h}^{n}\right\|\|\rho(h)\|_{L^{\infty}(\Omega)}|\Delta \rho(h)| \\
& \leq C k\left\|\boldsymbol{w}_{h}^{n}\right\|\|\rho(h)\|_{L^{\infty}(\Omega)}\left|\Delta_{h} \rho_{h}^{n+1}\right|,
\end{aligned}
$$

where $\boldsymbol{a} \otimes \boldsymbol{b}$ denotes the tensorial product matrix of two vectors $\boldsymbol{a}=\left(a_{i}\right)_{i=1}^{2}, \boldsymbol{b}=$ $\left(b_{i}\right)_{i=1}^{2}$, with coefficients $(\boldsymbol{a} \otimes \boldsymbol{b})_{i, j}=a_{i} b_{j}$. Accordingly,

$$
I \leq C k\left\|\boldsymbol{w}_{h}^{n}\right\|\left(\|\rho(h)\|_{L^{\infty}(\Omega)}+\left\|\nabla\left(\rho_{h}^{n+1}-\rho(h)\right)\right\|_{L^{3}(\Omega)}\right)\left|\Delta_{h} \rho_{h}^{n+1}\right| .
$$

Now we will prove the inequality:

$$
\left\|\nabla\left(\rho_{h}^{n+1}-\rho(h)\right)\right\|_{L^{3}(\Omega)} \leq C h^{1 / 2}|\rho(h)|_{H^{2}(\Omega)} .
$$

For this, we write

$$
\left\|\nabla\left(\rho_{h}^{n+1}-\rho(h)\right)\right\|_{L^{3}} \leq\left\|\nabla\left(\rho_{h}^{n+1}-I_{h} \rho(h)\right)\right\|_{L^{3}}+\left\|\nabla\left(I_{h} \rho(h)-\rho(h)\right)\right\|_{L^{3}} .
$$

By multiplying (4.4) by $\bar{\rho}_{h} \in W_{h}$ and subtracting to (4.1), one gets

$$
\left(\nabla \rho_{h}^{n+1}-\nabla \rho(h), \nabla \bar{\rho}_{h}\right)=0 \quad \forall \bar{\rho}_{h} \in W_{h} .
$$

By adding and subtracting $\nabla I_{h} \rho(h)$, and considering $\bar{\rho}_{h}=\rho_{h}^{n+1}-I_{h} \rho(h) \in W_{h}$, we obtain

$$
\begin{aligned}
\left|\nabla \rho_{h}^{n+1}-\nabla I_{h} \rho(h)\right|^{2} & =-\left(\nabla I_{h} \rho(h)-\nabla \rho(h), \nabla \rho_{h}^{n+1}-\nabla I_{h} \rho(h)\right) \\
& \leq\left|\nabla I_{h} \rho(h)-\nabla \rho(h)\right|\left|\nabla \rho_{h}^{n+1}-\nabla I_{h} \rho(h)\right|,
\end{aligned}
$$

whence

$$
\begin{gathered}
\left|\nabla \rho_{h}^{n+1}-\nabla I_{h} \rho(h)\right| \leq\left|\nabla I_{h} \rho(h)-\nabla \rho(h)\right| \leq C h|\rho(h)|_{H^{2}(\Omega)} \\
\left|\nabla \rho_{h}^{n+1}-\nabla \rho(h)\right| \leq C h|\rho(h)|_{H^{2}(\Omega)}
\end{gathered}
$$

Thus, by using the inverse inequality [7]

$$
\left\|\nabla \rho_{h}^{n+1}-\nabla I_{h} \rho(h)\right\|_{L^{3}(\Omega)} \leq C h^{-1 / 2}\left|\nabla \rho_{h}^{n+1}-\nabla I_{h} \rho(h)\right|
$$

Copyright $@$ by SIAM. Unauthorized reproduction of this article is prohibited. 
and (4.9), we arrive at

$$
\left\|\nabla \rho_{h}^{n+1}-\nabla I_{h} \rho(h)\right\|_{L^{3}(\Omega)} \leq C h^{1 / 2}|\rho(h)|_{H^{2}(\Omega)} .
$$

So, from (4.8) and (4.11) one gets (4.7) by taking into account the interpolation error $\left\|\nabla\left(\rho(h)-I_{h} \rho(h)\right)\right\|_{L^{3}(\Omega)} \leq C h^{1 / 2}|\rho(h)|_{H^{2}(\Omega)}$.

By getting back to (4.6) and using (4.7), we bound

$$
I \leq C k\left\|\boldsymbol{w}_{h}^{n}\right\|\left(\left\|\rho(h)-\rho_{h}^{n+1}\right\|_{L^{\infty}}+\left\|\rho_{h}^{n+1}\right\|_{L^{\infty}}+h^{1 / 2}|\rho(h)|_{H^{2}}\right)\left|\Delta_{h} \rho_{h}^{n+1}\right| .
$$

Now we write

$$
\left\|\rho_{h}^{n+1}-\rho(h)\right\|_{L^{\infty}(\Omega)} \leq\left\|\rho_{h}^{n+1}-I_{h} \rho(h)\right\|_{L^{\infty}(\Omega)}+\left\|I_{h} \rho(h)-\rho(h)\right\|_{L^{\infty}(\Omega)} .
$$

By using the interpolation error $\left\|\rho(h)-I_{h} \rho(h)\right\|_{L^{\infty}(\Omega)} \leq C h^{1 / 2}|\rho(h)|_{H^{2}(\Omega)}$, the inverse inequality in $3 \mathrm{D}$

$$
\begin{aligned}
\left\|\rho_{h}^{n+1}-I_{h} \rho(h)\right\|_{L^{\infty}(\Omega)} & \leq C h^{-1 / 2}\left\|\rho_{h}^{n+1}-I_{h} \rho(h)\right\|_{H^{1}(\Omega)} \\
& \leq C h^{-1 / 2}\left(\left\|\rho_{h}^{n+1}-\rho(h)\right\|_{H^{1}(\Omega)}+\left\|\rho(h)-I_{h} \rho(h)\right\|_{H^{1}(\Omega)}\right) \\
& \leq C h^{-1 / 2}\left(\left|\nabla\left(\rho_{h}^{n+1}-\rho(h)\right)\right|+h|\rho(h)|_{H^{2}(\Omega)}\right),
\end{aligned}
$$

where the generalized Poincare inequality has been used in the last line, since $\int_{\Omega} \rho_{h}^{n+1}$ $=\int_{\Omega} \rho_{h}^{0}$ (see Remark 3.1) and $\int_{\Omega} \rho_{h}^{0}=\int_{\Omega} \rho(h)$ (see (4.4)). By using (4.10) and (4.5),

$$
\left\|\rho_{h}^{n+1}-\rho(h)\right\|_{L^{\infty}(\Omega)} \leq C h^{1 / 2}|\rho(h)|_{H^{2}(\Omega)} \leq C h^{1 / 2}\left|\Delta_{h} \rho_{h}^{n+1}\right| .
$$

By applying the above estimate in (4.12), we bound

$$
\begin{aligned}
I & \leq C k\left\|\boldsymbol{w}_{h}^{n}\right\|\left(h^{1 / 2}\left|\Delta_{h} \rho_{h}^{n+1}\right|+\widetilde{M}\right)\left|\Delta_{h} \rho_{h}^{n+1}\right| \\
& \leq C k\left\|\boldsymbol{w}_{h}^{n}\right\| h^{1 / 2}\left|\Delta_{h} \rho_{h}^{n+1}\right|^{2}+\frac{C}{\lambda} k\left\|\boldsymbol{w}_{h}^{n}\right\|^{2}+\frac{\lambda}{2} k\left|\Delta_{h} \rho_{h}^{n+1}\right|^{2} .
\end{aligned}
$$

By Corollary 3.3 we infer the bound $\left\|\boldsymbol{w}_{h}^{n}\right\| \leq C / k^{1 / 2}$ (with $C$ independent of $\lambda, h, k$ ), and, by choosing $h$ and $k$ small enough such that

$$
C \sqrt{\frac{h}{k}} \leq \frac{1}{2} \lambda
$$

we get

$$
I \leq \frac{C}{\lambda} k\left\|\boldsymbol{w}_{h}^{n}\right\|^{2}+\lambda k\left|\Delta_{h} \rho_{h}^{n+1}\right|^{2} .
$$

Therefore, from (4.3) we get the inequality

$$
\left|\nabla \rho_{h}^{n+1}\right|^{2}-\left|\nabla \rho_{h}^{n}\right|^{2}+\left|\nabla\left(\rho_{h}^{n+1}-\rho_{h}^{n}\right)\right|^{2}+\lambda k\left|\Delta_{h} \rho_{h}^{n+1}\right|^{2} \leq C \frac{k}{\lambda}\left\|\boldsymbol{w}_{h}^{n}\right\|^{2} .
$$

By adding (4.13) for $n=0, \ldots, r$, with $r<N$, we arrive at

$$
\lambda\left|\nabla \rho_{h}^{r+1}\right|^{2}+\lambda \sum_{n=0}^{r}\left|\nabla\left(\rho_{h}^{n+1}-\rho_{h}^{n}\right)\right|^{2}+\lambda^{2} k \sum_{n=0}^{r}\left|\Delta_{h} \rho_{h}^{n+1}\right|^{2} \leq C k \sum_{n=0}^{r}\left\|\boldsymbol{w}_{h}^{n}\right\|^{2}+\lambda\left|\nabla \rho_{h}^{0}\right|^{2} .
$$

Copyright (c) by SIAM. Unauthorized reproduction of this article is prohibited. 
Finally, from estimate (ii) of Lemma 3.2, one gets the desired estimates (ix)-(xi).

COROLLARY 4.2. The following inequality holds:

$$
\left\|\nabla \rho_{h}^{n+1}\right\|_{L^{3}(\Omega)} \leq C\left|\nabla \rho_{h}^{n+1}\right|^{1 / 2}\left|\Delta_{h} \rho_{h}^{n+1}\right|^{1 / 2} .
$$

Consequently, under the hypotheses of Theorem 4.1, one has the estimate

$$
\lambda^{3} k \sum_{n=0}^{N-1}\left\|\rho_{h}^{n+1}\right\|_{W^{1,3}(\Omega)}^{4} \leq C,
$$

where $C>0$ is independent of $h, k$, and $\lambda$.

Proof. Thanks to estimates (ix) for $\left(\rho_{h}^{n}\right)$ and (x) for $\left(\Delta_{h} \rho_{h}^{n}\right)$, it suffices to prove (4.14). For this, by considering $\rho(h)$ the solution of problem (4.4), we have

$$
\left\|\nabla \rho_{h}^{n+1}\right\|_{L^{3}(\Omega)} \leq\left\|\nabla\left(\rho_{h}^{n+1}-\rho(h)\right)\right\|_{L^{3}(\Omega)}+\|\nabla \rho(h)\|_{L^{3}(\Omega)} .
$$

By using inequality (4.7) and the interpolation inequality

$$
\|\nabla \rho(h)\|_{L^{3}(\Omega)} \leq C|\nabla \rho(h)|^{1 / 2}\|\nabla \rho(h)\|_{H^{1}(\Omega)}^{1 / 2} \leq C|\nabla \rho(h)|^{1 / 2}|\rho(h)|_{H^{2}(\Omega)}^{1 / 2},
$$

we arrive at

$$
\left\|\nabla \rho_{h}^{n+1}\right\|_{L^{3}(\Omega)} \leq C h^{1 / 2}|\rho(h)|_{H^{2}(\Omega)}+C|\nabla \rho(h)|^{1 / 2}|\rho(h)|_{H^{2}(\Omega)}^{1 / 2} .
$$

Next, we bound the term $|\nabla \rho(h)|$ by using (4.10) as follows:

$$
|\nabla \rho(h)| \leq\left|\nabla\left(\rho(h)-\rho_{h}^{n+1}\right)\right|+\left|\nabla \rho_{h}^{n+1}\right| \leq C h|\rho(h)|_{H^{2}(\Omega)}+\left|\nabla \rho_{h}^{n+1}\right| .
$$

Therefore, by using (4.5)

$$
\begin{aligned}
\left\|\nabla \rho_{h}^{n+1}\right\|_{L^{3}(\Omega)} & \leq C h^{1 / 2}|\rho(h)|_{H^{2}(\Omega)}+C\left|\nabla \rho_{h}^{n+1}\right|^{1 / 2}|\rho(h)|_{H^{2}(\Omega)}^{1 / 2} \\
& \leq C h^{1 / 2}\left|\Delta_{h} \rho_{h}^{n+1}\right|+C\left|\nabla \rho_{h}^{n+1}\right|^{1 / 2}\left|\Delta_{h} \rho_{h}^{n+1}\right|^{1 / 2} .
\end{aligned}
$$

On the other hand, by considering $\bar{\rho}_{h}=-\Delta_{h} \rho_{h}^{n+1}$ in (4.1), we get

$$
\left|\Delta_{h} \rho_{h}^{n+1}\right|^{2} \leq\left|\nabla \rho_{h}^{n+1}\right|\left|\nabla \Delta_{h} \rho_{h}^{n+1}\right| \leq \frac{C}{h}\left|\nabla \rho_{h}^{n+1}\right|\left|\Delta_{h} \rho_{h}^{n+1}\right|,
$$

where we have used the inverse inequality between $L^{2}$ and $H^{1}$. The last two estimates imply (4.14).

5. Weak convergence. To study the convergence of scheme (1.8)-(1.10), we define the following functions.

Definition 5.1. One defines $\boldsymbol{u}_{h, k}$ (respectively, $\widehat{\boldsymbol{u}}_{h, k}, \widehat{\boldsymbol{w}}_{h, k}$, and $p_{h, k}$ ) as the piecewise constant functions taking values $\boldsymbol{u}_{h}^{n+1}$ on $\left(t_{n}, t_{n+1}\right]$ (respectively, $\boldsymbol{u}_{h}^{n}, \boldsymbol{w}_{h}^{n}$, and $\left.p_{h}^{n+1}\right)$. Analogously, we define $\rho_{h, k}$ and $\widehat{\rho}_{h, k}$. Moreover, one defines $\widetilde{\rho}_{h, k} \in$ $C^{0}\left([0, T] ; \boldsymbol{V}_{h}\right)$ as the piecewise linear functions such that $\widetilde{\rho}_{h, k}\left(t_{n}\right)=\rho_{h}^{n}$.

Lemma 3.2, Corollary 3.3, Theorem 3.5, Theorem 4.1, and Corollary 4.2 imply the following estimates (independent of $h$ and $k$, but some of them depend on $\lambda$ ):

$$
\begin{gathered}
\left\{\boldsymbol{u}_{h, k}\right\}_{h, k},\left\{\widehat{\boldsymbol{u}}_{h, k}\right\}_{h, k},\left\{\widehat{\boldsymbol{w}}_{h, k}\right\}_{h, k} \text { in } L^{\infty}\left(0, T ; \boldsymbol{L}^{2}(\Omega)\right) \cap L^{2}\left(0, T ; \boldsymbol{H}_{0}^{1}(\Omega)\right), \\
\left\{\widetilde{\rho}_{h, k}\right\}_{h, k},\left\{\rho_{h, k}\right\}_{h, k},\left\{\widehat{\rho}_{h, k}\right\}_{h, k} \text { in } L^{\infty}\left(0, T ; H^{1}(\Omega)\right) \cap L^{\infty}(Q), \\
\left\{\rho_{h, k}\right\}_{h, k} \text { in } L^{4}\left(0, T ; W^{1,3}(\Omega)\right) .
\end{gathered}
$$

Copyright $\odot$ by SIAM. Unauthorized reproduction of this article is prohibited. 
Now let us pass to the limit in both discrete free-divergence equations (1.10) and (3.1). Consider $q \in C^{0}\left([0, T] ; C^{\infty}(\Omega)\right)$ such that $\int_{\Omega} q(\boldsymbol{x}) d \boldsymbol{x}=0, q_{h}^{n}=K_{h} q\left(t_{n}\right) \in M_{h}$, and $\bar{q}_{h}^{n}=\widetilde{K}_{h} q\left(t_{n}\right) \in \widetilde{M}_{h}$. Define $q_{h, k}$ and $\bar{q}_{h, k}$ as $p_{h, k}$ in Definition 5.1. On the other hand, we know that there exist two limit functions $\boldsymbol{w}$ and $\boldsymbol{u}$ belonging to $\boldsymbol{H}_{0}^{1}(\Omega)$ such that $\boldsymbol{u}_{h, k} \rightarrow \boldsymbol{u}$ and $\boldsymbol{w}_{h, k} \rightarrow \boldsymbol{w}$ weakly in $L^{2}\left(0, T ; \boldsymbol{H}_{0}^{1}(\Omega)\right)$ as $(h, k) \rightarrow 0$. Thus, we write from (1.10)

$$
0=k \sum_{n=1}^{N}\left(\nabla \cdot \boldsymbol{u}_{h}^{n}, q_{h}^{n}\right)=\int_{0}^{T}\left(\nabla \cdot \boldsymbol{u}_{h, k}, q_{h, k}\right) d t \rightarrow \int_{0}^{T}(\nabla \cdot \boldsymbol{u}, q) d t=0
$$

for all $q \in C^{0}\left([0, T] ; C^{\infty}(\Omega)\right)$, with $\int_{\Omega} q(\boldsymbol{x}) d \boldsymbol{x}=0$. A density argument says $\boldsymbol{u} \in \boldsymbol{V}$. In an analogous way, we can also prove that $\boldsymbol{w} \in \boldsymbol{V}$.

Next, we wish to derive a test function for (1.7), a discrete free-divergence approximation of a function $\boldsymbol{v} \in \boldsymbol{C}_{c}^{\infty}(\Omega)$, with $\nabla \cdot \boldsymbol{v}=0$.

Lemma 5.2. Let $\overline{\boldsymbol{w}} \in \boldsymbol{C}_{c}^{\infty}(\Omega)$. Then there exists $\overline{\boldsymbol{w}}_{h} \in \widetilde{\boldsymbol{V}}_{h}$ such that:

$$
\overline{\boldsymbol{w}}_{h} \rightarrow \overline{\boldsymbol{w}} \quad \text { in } \boldsymbol{H}_{0}^{1}(\Omega) \quad \text { and } \quad\left(\nabla \cdot \overline{\boldsymbol{w}}_{h}, \bar{q}_{h}\right)=\left(\nabla \cdot \overline{\boldsymbol{w}}, \bar{q}_{h}\right) \forall \bar{q}_{h} \in \widetilde{M}_{h} .
$$

A proof of this result can be found in [11].

By taking into account previous arguments, we can arrive at the following result.

LEMma 5.3. Under the hypotheses of Theorem 3.5 there exist subsequences of $\left\{\boldsymbol{u}_{h, k}\right\}_{h, k},\left\{\widehat{\boldsymbol{u}}_{h, k}\right\}_{h, k},\left\{\widehat{\boldsymbol{w}}_{h, k}\right\}_{h, k},\left\{\rho_{h, k}\right\}_{h, k, \lambda},\left\{\widehat{\rho}_{h, k}\right\}_{h, k}$, and $\left\{\widetilde{\rho}_{h, k}\right\}_{h, k}$ (denoted in the same way) and limit functions $\boldsymbol{u}, \rho$ verifying the following weak convergences as $(h, k) \rightarrow 0$ :

$$
\begin{array}{r}
\boldsymbol{u}_{h, k} \rightarrow \boldsymbol{u}, \quad \widehat{\boldsymbol{u}}_{h, k} \rightarrow \boldsymbol{u}, \quad \widehat{\boldsymbol{w}}_{h, k} \rightarrow \boldsymbol{u} \quad \text { in }\left\{\begin{array}{l}
L^{2}\left(0, T ; \boldsymbol{H}_{0}^{1}(\Omega)\right) \text {-weak }, \\
L^{\infty}\left(0, T ; \boldsymbol{L}^{2}(\Omega)\right) \text {-weak* },
\end{array}\right. \\
\rho_{h, k} \rightarrow \rho, \quad \widehat{\rho}_{h, k} \rightarrow \rho, \quad \widehat{\rho}_{h, k} \rightarrow \rho \quad \text { in }\left\{\begin{array}{l}
L^{\infty}(Q) \text {-weak } *, \\
L^{\infty}\left(0, T ; H^{1}(\Omega)\right) \text {-weak } *
\end{array}\right. \\
\widehat{\rho}_{h, k} \rightarrow \rho \text { in } L^{4}\left(0, T ; W^{1,3}(\Omega)\right) \text {-weak } .
\end{array}
$$

Proof. Let us prove only that $\widehat{\boldsymbol{w}}_{h, k} \rightarrow \boldsymbol{u}$ in $L^{2}\left(0, T ; \boldsymbol{H}_{0}^{1}(\Omega)\right.$-weak. Consider $\boldsymbol{v} \in C^{0}\left([0, T] ; \boldsymbol{C}_{c}^{\infty}(\Omega)\right)$, with $\nabla \cdot \boldsymbol{v}=0$ and $\boldsymbol{v}_{h}^{n} \in \widetilde{\boldsymbol{V}}_{h}$ an approximation of $\boldsymbol{v}\left(t_{n}\right)$ given by Lemma 5.2. Define $\boldsymbol{v}_{h, k} \in L^{\infty}\left(0, T ; \widetilde{\boldsymbol{V}}_{h}\right)$ as the piecewise constant functions taking values $\boldsymbol{v}_{h}^{n+1}$ on $\left(t_{n}, t_{n+1}\right]$ which verifies $\boldsymbol{v}_{h, k} \rightarrow \boldsymbol{v}$ in $L^{\infty}\left(0, T ; \boldsymbol{H}_{0}^{1}(\Omega)\right)$. Next, by testing (1.7) by the test function $\boldsymbol{v}_{h}^{n}$, we have $\left(\nabla\left(\boldsymbol{w}_{h}^{n}-\boldsymbol{u}_{h}^{n}\right), \nabla \boldsymbol{v}_{n}^{n}\right)=0$, since the pressure term vanishes. By multiplying by the time step $k$, summing over $n$, and passing to limit as $(h, k)$ tend to zero, we infer that

$$
\int_{0}^{T}(\nabla(\boldsymbol{w}-\boldsymbol{u}), \nabla \boldsymbol{v})=0 \quad \forall \boldsymbol{v} \in C^{0}\left([0, T] ; \boldsymbol{C}_{c}^{\infty}(\Omega)\right), \text { with } \nabla \cdot \boldsymbol{v}=0 .
$$

A density argument provides that this equality holds for any $\boldsymbol{v} \in L^{2}(0, T ; \boldsymbol{V})$. Therefore, we can choose $\boldsymbol{v}=\boldsymbol{w}-\boldsymbol{u}$ ( since $\nabla \cdot \boldsymbol{u}=0$ and $\nabla \cdot \boldsymbol{w}=0$ ); then $\boldsymbol{w}=\boldsymbol{u}$.

6. Strong convergence. As usual for nonlinear systems, strong convergence in some suitable space is necessary to identify the limit of the nonlinear terms. 


\subsection{Strong convergence for the density in $L^{2}(\Omega)$.}

Lemma 6.1. Under the hypotheses of Theorem 4.1, one has:

$$
k \sum_{n=0}^{N}\left|\frac{\rho_{h}^{n+1}-\rho_{h}^{n}}{k}\right|^{4 / 3} \leq C_{\lambda}
$$

where $C_{\lambda}>0$ is independent of $h$ and $k$ (but depends on $\lambda$ ).

Proof. We consider $P_{h}: L^{2}(\Omega) \rightarrow W_{h}$ the orthogonal projector, defined as $\left(P_{h} w-\right.$ $\left.w, w_{h}\right)=0$ for any $w_{h} \in W_{h}$. Let $w \in L^{2}(\Omega)$. By taking in (1.8) as a test function $w_{h}=P_{h} w$, we arrive at

$$
\left(\frac{\rho_{h}^{n+1}-\rho_{h}^{n}}{k}, w\right)+\left(\boldsymbol{w}_{h}^{n} \cdot \nabla \rho_{h}^{n+1}, P_{h} w\right)-\lambda\left(\Delta_{h} \rho_{h}^{n+1}, w\right)=0
$$

where we have used the definition of $P_{h}$ in the first and last terms. By taking into account the stability of the projector operator $\left|P_{h} w\right| \leq|w|$, we get

$$
\left|\frac{\rho_{h}^{n+1}-\rho_{h}^{n}}{k}\right| \leq\left\|\boldsymbol{w}_{h}^{n}\right\|_{L^{6}(\Omega)}\left\|\nabla \rho_{h}^{n+1}\right\|_{L^{3}(\Omega)}+\lambda\left|\Delta_{h} \rho_{h}^{n+1}\right| .
$$

By summing up over $n$ and using the estimates of $\left\{\widehat{\boldsymbol{w}}_{h, k}\right\}_{h, k}$ in $L^{2}\left(0, T ; \boldsymbol{L}^{6}(\Omega)\right.$ ) (due to the estimates in $L^{2}\left(0, T ; \boldsymbol{H}^{1}(\Omega)\right)$ ), of $\left\{\lambda \Delta_{h} \rho_{h, k}\right\}_{h, k}$ in $L^{2}\left(0, T ; L^{2}(\Omega)\right)$, and of $\left\{\nabla \rho_{h, k}\right\}_{h, k}$ in $L^{4}\left(0, T ; \boldsymbol{L}^{3}(\Omega)\right.$ ) (this last estimate depends on $\lambda$ ), we can conclude the result.

Remark 6.2. As a consequence of the previous lemma, the estimate

$$
\left\|\frac{d}{d t} \widetilde{\rho}_{h, k}\right\|_{L^{4 / 3}\left(0, T ; L^{2}(\Omega)\right)} \leq C_{\lambda}
$$

holds. On the other hand, from Lemma 3.2 , we get $\left\|\widetilde{\rho}_{h, k}\right\|_{L^{\infty}\left(0, T ; H^{1}(\Omega)\right)} \leq C_{\lambda}$. Then, thanks to an Aubin-Lions compactness argument, one has $\widetilde{\rho}_{h, k} \rightarrow \rho$ in $L^{\infty}\left(0, T ; L^{p}(\Omega)\right)$ as $(h, k) \rightarrow 0$, with $p<6$. From this convergence, we deduce that $\rho_{h, k}, \widehat{\rho}_{h, k} \rightarrow \rho$ in $L^{2}\left(0, T ; L^{2}(\Omega)\right)$ as $(h, k) \rightarrow 0$, since

$$
\left\|\widetilde{\rho}_{h, k}-\rho_{k, h}\right\|_{L^{2}\left(0, T ; L^{2}(\Omega)\right)}^{2} \leq\left\|\widehat{\rho}_{h, k}-\rho_{k, h}\right\|_{L^{2}\left(0, T ; L^{2}(\Omega)\right)}^{2}=k \sum_{n=0}^{N-1}\left|\rho_{h}^{n+1}-\rho_{h}^{n}\right|^{2} \leq C k .
$$

\subsection{Strong convergence for the velocity.}

Proposition 6.3. Under the hypotheses of Theorem 4.1, the following estimate holds:

$$
\int_{0}^{T-\delta}\left|\sqrt{\rho_{h, k}(t+\delta)}\left(\boldsymbol{u}_{h, k}(t+\delta)-\boldsymbol{u}_{h, k}(t)\right)\right|^{2} d t \leq C_{\lambda} \delta^{1 / 4} \quad \forall \delta: \quad 0<\delta<T,
$$

where $C_{\lambda}>0$ is independent of $h, k$, and $\delta$ (but depends on $\lambda$ ).

Proof. Throughout the proof we will keep in mind Lemmas 3.2 and 4.1 and Remark 3.3. As $\rho_{h, k}$ and $\boldsymbol{u}_{h, k}$ are piecewise constant functions, it suffices to suppose that $\delta$ is proportional to the time step $k$, i.e., $\delta=r k$ for any $r=0, \ldots, N$. Then, to obtain (6.1), it suffices to prove that

$$
k \sum_{m=0}^{N-r}\left|\sqrt{\rho_{h}^{m+r}}\left(\boldsymbol{u}_{h}^{m+r}-\boldsymbol{u}_{h}^{m}\right)\right|^{2} \leq C_{\lambda}(r k)^{1 / 4} \quad \forall r: 0 \leq r \leq N .
$$

Copyright (c) by SIAM. Unauthorized reproduction of this article is prohibited. 
Let us write the time derivative of the discrete momentum system (1.9) in conservative form. By adding at the right- and left-hand sides of (1.9) the term

$$
\begin{aligned}
& \frac{1}{2}\left(\frac{\rho_{h}^{n+1}-\rho_{h}^{n}}{k}, \boldsymbol{u}_{h}^{n+1} \cdot \overline{\boldsymbol{u}}_{h}\right): \\
& \left\{\begin{array}{l}
\left(\frac{\rho_{h}^{n+1} \boldsymbol{u}_{h}^{n+1}-\rho_{h}^{n} \boldsymbol{u}_{h}^{n}}{k}, \overline{\boldsymbol{u}}_{h}\right)+a\left(\rho_{h}^{n+1}, \boldsymbol{u}_{h}^{n+1}, \overline{\boldsymbol{u}}_{h}\right)-\left(p_{h}^{n+1}, \nabla \cdot \overline{\boldsymbol{u}}_{h}\right) \\
+c\left(\rho_{h}^{n+1} \boldsymbol{u}_{h}^{n}-\lambda \nabla \rho_{h}^{n+1}, \boldsymbol{u}_{h}^{n+1}, \overline{\boldsymbol{u}}_{h}\right)=\left(\rho_{h}^{n+1} \boldsymbol{f}^{n+1}, \overline{\boldsymbol{u}}_{h}\right)+\frac{1}{2}\left(\frac{\rho_{h}^{n+1}-\rho_{h}^{n}}{k}, \boldsymbol{u}_{h}^{n+1} \cdot \overline{\boldsymbol{u}}_{h}\right) .
\end{array}\right.
\end{aligned}
$$

By multiplying (6.3) by $k$ and summing for $n=m, \ldots, m-1+r$, we have

$$
\left\{\begin{array}{l}
\left(\rho_{h}^{m+r} \boldsymbol{u}_{h}^{m+r}-\rho_{h}^{m} \boldsymbol{u}_{h}^{m}, \overline{\boldsymbol{u}}_{h}\right)+k \sum_{n=m}^{m-1+r} a\left(\rho_{h}^{n+1}, \boldsymbol{u}_{h}^{n+1}, \overline{\boldsymbol{u}}_{h}\right)-\sum_{n=m}^{m-1+r}\left(p_{h}^{n+1}, \nabla \cdot \overline{\boldsymbol{u}}_{h}\right) \\
+k \sum_{n=m}^{m-1+r} c\left(\rho_{h}^{n+1} \boldsymbol{u}_{h}^{n}-\lambda \nabla \rho_{h}^{n+1}, \boldsymbol{u}_{h}^{n+1}, \overline{\boldsymbol{u}}_{h}\right) \\
=k \sum_{n=m}^{m-1+r}\left(\rho_{h}^{n+1} \boldsymbol{f}^{n+1}, \overline{\boldsymbol{u}}_{h}\right)+\frac{k}{2} \sum_{n=m}^{m-1+r}\left(\frac{\rho_{h}^{n+1}-\rho_{h}^{n}}{k}, \boldsymbol{u}_{h}^{n+1} \cdot \overline{\boldsymbol{u}}_{h}\right) .
\end{array}\right.
$$

By taking $\overline{\boldsymbol{u}}_{h}=\boldsymbol{u}_{h}^{m+r}-\boldsymbol{u}_{h}^{m}$ and making use of the identity

$$
\rho_{h}^{m+r} \boldsymbol{u}_{h}^{m+r}-\rho_{h}^{m} \boldsymbol{u}_{h}^{m}=\rho_{h}^{m+r}\left(\boldsymbol{u}_{h}^{m+r}-\boldsymbol{u}_{h}^{m}\right)+\left(\rho_{h}^{m+r}-\rho_{h}^{m}\right) \boldsymbol{u}_{h}^{m},
$$

we get

$$
\left\{\begin{array}{l}
\left|\sqrt{\rho_{h}^{m+r}}\left(\boldsymbol{u}_{h}^{m+r}-\boldsymbol{u}_{h}^{m}\right)\right|^{2}=-\left(\rho_{h}^{m+r}-\rho_{h}^{m}, \boldsymbol{u}_{h}^{m} \cdot\left(\boldsymbol{u}_{h}^{m+r}-\boldsymbol{u}_{h}^{m}\right)\right) \\
-k \sum_{n=m}^{m-1+r}\left\{a\left(\rho_{h}^{n+1}, \boldsymbol{u}_{h}^{n+1}, \boldsymbol{u}_{h}^{m+r}-\boldsymbol{u}_{h}^{m}\right)+c\left(\rho_{h}^{n+1} \boldsymbol{u}_{h}^{n}-\lambda \nabla \rho_{h}^{n+1}, \boldsymbol{u}_{h}^{n+1}, \boldsymbol{u}_{h}^{m+r}-\boldsymbol{u}_{h}^{m}\right)\right\} \\
+k \sum_{n=m}^{m-1+r}\left\{\left(\rho_{h}^{n+1} \boldsymbol{f}^{n+1}, \boldsymbol{u}_{h}^{m+r}-\boldsymbol{u}_{h}^{m}\right)+\frac{1}{2}\left(\frac{\rho_{h}^{n+1}-\rho_{h}^{n}}{k}, \boldsymbol{u}_{h}^{n+1} \cdot\left(\boldsymbol{u}_{h}^{m+r}-\boldsymbol{u}_{h}^{m}\right)\right)\right\} .
\end{array}\right.
$$

On the other hand, by taking $\bar{\rho}_{h}=\rho_{h}^{m+r}-\rho_{h}^{m}$ as a test function in the density scheme (4.2), multiplying by $k$, and summing for $n=m, \ldots, m-1+r$, we obtain

$$
\begin{aligned}
\left|\rho_{h}^{m+r}-\rho_{h}^{m}\right|^{2} & =-k \sum_{n=m}^{m-1+r}\left(\boldsymbol{w}_{h}^{n} \cdot \nabla \rho_{h}^{n+1}-\lambda \Delta_{h} \rho_{h}^{n+1}, \rho_{h}^{m}-\rho_{h}^{m+r}\right) \\
& \leq k \sum_{n=m}^{m-1+r}\left(\left\|\boldsymbol{w}_{h}^{n}\right\|_{\boldsymbol{L}^{6}(\Omega)}\left\|\nabla \rho_{h}^{n}\right\|_{\boldsymbol{L}^{3}(\Omega)}+\lambda\left|\Delta_{h} \rho_{h}^{n+1}\right|\right)\left|\rho_{h}^{m}-\rho_{h}^{m+r}\right| .
\end{aligned}
$$

Therefore,

$$
\begin{aligned}
\left|\rho_{h}^{m}-\rho_{h}^{m+r}\right| & \leq C\left(k \sum_{n=m}^{m-1+r}\left\|\boldsymbol{w}_{h}^{n}\right\|_{\boldsymbol{L}^{6}(\Omega)}^{2}\right)^{1 / 2}\left(k \sum_{n=m}^{m-1+r}\left\|\nabla \rho_{h}^{n}\right\|_{\boldsymbol{L}^{3}(\Omega)}^{4}\right)^{1 / 4}(r k)^{1 / 4} \\
& +C\left(k \sum_{n=m}^{m-1+r} \lambda\left|\Delta_{h} \rho_{h}^{n+1}\right|^{2}\right)^{1 / 2}(r k)^{1 / 2} \leq C_{\lambda}(r k)^{1 / 4}+C(r k)^{1 / 2}
\end{aligned}
$$

Copyright $\odot$ by SIAM. Unauthorized reproduction of this article is prohibited. 
where $C_{\lambda}>0$ is a constant independent of $h$ and $k$ (and depending on $\lambda$ ). Then we have

$$
\max _{1 \leq m \leq N}\left|\rho_{h}^{m}-\rho_{h}^{m+r}\right| \leq C_{\lambda}(r k)^{1 / 4} .
$$

By multiplying (6.5) by $k$ and summing for $m=0, \ldots, N-r$, we are going to get the desired bound (6.2) using (6.6). Indeed, from (6.6), one can obtain (with a similar argument as in [11])

$$
-k \sum_{m=0}^{N-r}\left(\rho_{h}^{m}-\rho_{h}^{m+r}, \boldsymbol{u}_{h}^{m} \cdot\left(\boldsymbol{u}_{h}^{m+r}-\boldsymbol{u}_{h}^{m}\right)\right) \leq C_{\lambda}(r k)^{1 / 4} .
$$

We analyze only the two terms whose estimates will be different from the ones done in [11]:

$$
\begin{aligned}
J_{1} & :=-k^{2} \sum_{m=0}^{N-r} \sum_{n=m}^{m-1+r} c\left(\rho_{h}^{n+1} \boldsymbol{u}_{h}^{n}-\lambda \nabla \rho_{h}^{n+1}, \boldsymbol{u}_{h}^{n+1}, \boldsymbol{u}_{h}^{m+r}-\boldsymbol{u}_{h}^{m}\right), \\
J_{2} & :=\frac{k^{2}}{2} \sum_{m=0}^{N-r} \sum_{n=m}^{m-1+r}\left(\frac{\rho_{h}^{n+1}-\rho_{h}^{n}}{k}, \boldsymbol{u}_{h}^{n+1} \cdot\left(\boldsymbol{u}_{h}^{m+r}-\boldsymbol{u}_{h}^{m}\right)\right) .
\end{aligned}
$$

To estimate $J_{1}$, we use (1.11) as follows:

$$
\begin{aligned}
J_{1} & \leq C k^{2} \sum_{m=0}^{N-r} \sum_{n=m}^{m-1+r}\left\|\rho_{h}^{n+1} \boldsymbol{u}_{h}^{n}-\lambda \nabla \rho_{h}^{n+1}\right\|_{\boldsymbol{L}^{3}(\Omega)}\left\|\boldsymbol{u}_{h}^{n+1}\right\|\left\|\boldsymbol{u}_{h}^{m+r}-\boldsymbol{u}_{h}^{m}\right\| \\
& \leq C k^{2} \sum_{m=0}^{N-r} \sum_{n=m}^{m-1+r}\left(\left\|\rho_{h}^{n+1}\right\|_{L^{\infty}(\Omega)}\left\|\boldsymbol{u}_{h}^{n}\right\|_{\boldsymbol{L}^{3}(\Omega)}+\lambda\left\|\nabla \rho_{h}^{n+1}\right\|_{\boldsymbol{L}^{3}(\Omega)}\right)\left\|\boldsymbol{u}_{h}^{n+1}\right\|\left\|\boldsymbol{u}_{h}^{m+r}-\boldsymbol{u}_{h}^{m}\right\| .
\end{aligned}
$$

By interchanging the sum order (Fubini's discrete rule) and using the fact that $\left\|\rho_{h}^{n+1}\right\|_{L^{\infty}(\Omega)} \leq C$

$$
J_{1} \leq C k^{2} \sum_{n=0}^{N-1}\left(\left\|\boldsymbol{u}_{h}^{n}\right\|_{\boldsymbol{L}^{3}(\Omega)}+\lambda\left\|\nabla \rho_{h}^{n+1}\right\|_{\boldsymbol{L}^{3}(\Omega)}\right)\left\|\boldsymbol{u}_{h}^{n+1}\right\| \sum_{m=\overline{n-r+1}}^{\bar{n}}\left\|\boldsymbol{u}_{h}^{m+r}-\boldsymbol{u}_{h}^{m}\right\|,
$$

where

$$
\bar{n}= \begin{cases}0 & \text { if } \quad n<0 \\ n & \text { if } \quad 0 \leq n \leq N-r \\ N-r & \text { if } \quad n>N-r\end{cases}
$$

Next, by taking into account that $|\bar{n}-\overline{n-r+1}| \leq r$ and Corollary 4.2, we get

$$
\begin{aligned}
J_{1} \leq & C k \sum_{n=0}^{N-1}\left(\left\|\boldsymbol{u}_{h}^{n}\right\|_{L^{3}(\Omega)}+\lambda\left\|\nabla \rho_{h}^{n+1}\right\|_{L^{3}(\Omega)}\right)\left\|\boldsymbol{u}_{h}^{n+1}\right\| \\
& \left(\sum_{m=\overline{n-r+1}}^{\bar{n}} k\left\|\boldsymbol{u}_{h}^{m+r}-\boldsymbol{u}_{h}^{m}\right\|^{2}\right)^{1 / 2}\left(\sum_{m=\overline{n-r+1}}^{\bar{n}} k\right)^{1 / 2} \\
\leq & C(r k)^{1 / 2}\left(k \sum_{n=0}^{N-1}\left(\left\|\boldsymbol{u}_{h}^{n}\right\|_{L^{3}(\Omega)}+\lambda\left\|\nabla \rho_{h}^{n+1}\right\|_{L^{3}(\Omega)}\right)^{2}\right)^{1 / 2}\left(k \sum_{n=0}^{N-1}\left\|\boldsymbol{u}_{h}^{n+1}\right\|^{2}\right)^{1 / 2} \\
\leq & C(r k)^{1 / 2} .
\end{aligned}
$$

In the same way, we can bound the term $k^{2} \sum_{m=0}^{N-r} \sum_{n=m}^{m-1+r} a\left(\rho_{h}^{n+1}, \boldsymbol{u}_{h}^{n+1}, \boldsymbol{u}_{h}^{m+r}-\boldsymbol{u}_{h}^{m}\right)$. 
We bound $J_{2}$ as follows:

$$
\begin{aligned}
J_{2} & \leq C k^{2} \sum_{m=0}^{N-r} \sum_{n=m}^{m-1+r}\left|\frac{\rho_{h}^{n+1}-\rho_{h}^{n}}{k}\right|\left\|\boldsymbol{u}_{h}^{n+1}\right\|_{L^{3}(\Omega)}\left\|\boldsymbol{u}_{h}^{m+r}-\boldsymbol{u}_{h}^{m}\right\| \\
\text { (Fubini) } & \leq C k^{2} \sum_{n=0}^{N-1}\left|\frac{\rho_{h}^{n+1}-\rho_{h}^{n}}{k}\right|\left\|\boldsymbol{u}_{h}^{n+1}\right\|^{1 / 2}\left|\boldsymbol{u}_{h}^{n+1}\right|^{1 / 2} \sum_{m=\overline{n-r+1}}^{\bar{n}}\left\|\boldsymbol{u}_{h}^{m+r}-\boldsymbol{u}_{h}^{m}\right\| \\
& \leq C k \sum_{n=0}^{N-1}\left|\frac{\rho_{h}^{n+1}-\rho_{h}^{n}}{k}\right|\left\|\boldsymbol{u}_{h}^{n+1}\right\|^{1 / 2}\left(k \sum_{m=\overline{n-r+1}}^{\bar{n}}\left\|\boldsymbol{u}_{h}^{m+r}-\boldsymbol{u}_{h}^{m}\right\|^{2}\right)^{1 / 2}(r k)^{1 / 2} \\
& \leq C(r k)^{1 / 2}\left(k \sum_{n=0}^{N-1}\left|\frac{\rho_{h}^{n+1}-\rho_{h}^{n}}{k}\right|^{4 / 3}\right)^{3 / 4}\left(k \sum_{n=0}^{N-1}\left\|\boldsymbol{u}_{h}^{n+1}\right\|^{2}\right)^{1 / 4} \\
& \leq C_{\lambda}(r k)^{1 / 2} . \quad \square
\end{aligned}
$$

Remark 6.4. From the weak estimates of the discrete velocity $\boldsymbol{u}_{h, k}$ in $L^{\infty}(0, T$; $\left.\boldsymbol{L}^{2}(\Omega)\right) \cap L^{2}\left(0, T ; \boldsymbol{H}_{0}^{1}(\Omega)\right)$ and the fractional in time estimate of $\boldsymbol{u}_{h, k}$ given in (6.1), we can apply a compactness result [17] and obtain $\boldsymbol{u}_{h, k} \rightarrow \boldsymbol{u}$ in $L^{2}\left(0, T ; \boldsymbol{L}^{2}(\Omega)\right)$-strong. Consequently, thanks to estimate (iii), $\widehat{\boldsymbol{u}}_{h, k} \rightarrow \boldsymbol{u}$ in $L^{2}\left(0, T ; \boldsymbol{L}^{2}(\Omega)\right)$-strong. Finally, since $\left\{\boldsymbol{u}_{h, k}\right\}_{h, k}$ is bounded in $L^{\infty}\left(0, T ; \boldsymbol{L}^{2}(\Omega)\right)$ we improve the compactness of $\left\{\boldsymbol{u}_{h, k}\right\}_{h, k}$ to the $L^{p}\left(0, T ; \boldsymbol{L}^{2}(\Omega)\right)$ space, with $p<\infty$.

6.3. Strong convergence for the density in $H^{1}(\Omega)$. By using the compactness of the discrete density in $L^{2}\left(0, T ; \boldsymbol{L}^{2}(\Omega)\right)$ and comparing the equation for the discrete Laplacian and its limit (see [11]), one can obtain the convergence of the $L^{2}\left(0, T ; \boldsymbol{L}^{2}(\Omega)\right)$-norm of $\nabla \rho_{h, k}$ towards the same norm of $\nabla \rho$. Consequently, one has

$$
\left\|\rho_{h, k}-\rho\right\|_{L^{2}\left(0, T ; H^{1}(\Omega)\right)} \rightarrow 0 \text { as }(h, k) \rightarrow 0 .
$$

\section{Passing to the limit.}

7.1. Convergence for the density scheme. Thanks to the previous convergences, we can prove [11] the convergence of the density scheme as $(h, k) \rightarrow 0$, obtaining

$$
\rho_{t}+\boldsymbol{u} \cdot \nabla \rho-\lambda \Delta \rho=0 \text { in } Q,\left.\quad \frac{\partial \rho}{\partial \boldsymbol{n}}\right|_{\Sigma}=0, \quad \rho(0)=\rho_{0} \text { in } \Omega .
$$

7.2. Convergence for the momentum scheme. We use the following convergence result, which is similar to Lemma 5.2.

Lemma 7.1. Let $\overline{\boldsymbol{u}} \in \boldsymbol{C}_{c}^{\infty}(\Omega)$. Then there exists $\overline{\boldsymbol{u}}_{h} \in \boldsymbol{V}_{h}$ such that:

$$
\overline{\boldsymbol{u}}_{h} \rightarrow \overline{\boldsymbol{u}} \text { in } \boldsymbol{H}_{0}^{1}(\Omega) \quad \text { and } \quad\left(\nabla \cdot \overline{\boldsymbol{u}}_{h}, q_{h}\right)=\left(\nabla \cdot \overline{\boldsymbol{u}}, q_{h}\right) \forall q_{h} \in M_{h} .
$$

To pass to the limit in the discrete momentum system, we consider $\boldsymbol{v} \in C^{1}([0, T]$; $\mathbf{C}_{c}^{\infty}(\Omega)$ ), with $\nabla \cdot \boldsymbol{v}=0$ and $\boldsymbol{v}(T)=0$. We define $\boldsymbol{v}_{h}^{n}$ as the projection of $\boldsymbol{v}\left(t^{n}\right)$ furnished by Lemma 7.1. We define $\boldsymbol{v}_{h, k} \in L^{\infty}\left(0, T ; \boldsymbol{V}_{h}\right)$ as the piecewise constant functions taking values $\boldsymbol{v}_{h}^{n+1}$ on $\left(t_{n}, t_{n+1}\right]$ and let $\widetilde{\boldsymbol{v}}_{h, k} \in C^{0}\left([0, T] ; \boldsymbol{V}_{h}\right)$ be the piecewise linear, globally continuous functions and such that $\widetilde{\boldsymbol{v}}_{h, k}\left(t_{n}\right)=\boldsymbol{v}_{h}^{n}$. It is known that, as $(h, k) \rightarrow 0$,

$$
\boldsymbol{v}_{h, k} \rightarrow \boldsymbol{v} \quad \text { in } L^{\infty}\left(0, T ; \boldsymbol{H}_{0}^{1}(\Omega)\right), \quad \widetilde{\boldsymbol{v}}_{h, k} \rightarrow \boldsymbol{v} \quad \text { in } W^{1, \infty}\left(0, T ; \boldsymbol{H}_{0}^{1}(\Omega)\right) .
$$


By taking $\overline{\boldsymbol{u}}_{h}=\boldsymbol{v}_{h}^{n+1}$ as a test function in (6.3), multiplying by $k$, summing over $n$, and using the expression (discrete integration by parts in time)

$\sum_{n=0}^{N-1}\left(\rho_{h}^{n+1} \boldsymbol{u}_{h}^{n+1}-\rho_{h}^{n} \boldsymbol{u}_{h}^{n}, \boldsymbol{v}_{h}^{n+1}\right)=\left(\rho_{h}^{N} \boldsymbol{u}_{h}^{N}, \boldsymbol{v}_{h}^{N}\right)-\sum_{n=0}^{N-1}\left(\rho_{h}^{n} \boldsymbol{u}_{h}^{n}, \boldsymbol{v}_{h}^{n+1}-\boldsymbol{v}_{h}^{n}\right)-\left(\rho_{0 h} \boldsymbol{u}_{0 h}, \boldsymbol{v}_{h}^{0}\right)$

and the fact that $\boldsymbol{v}_{h}^{N}=0$ (since $\left.\boldsymbol{v}(T)=0\right)$, the following "conservative" formulation holds:

$$
\left\{\begin{array}{l}
-k \sum_{n=0}^{N-1}\left(\rho_{h}^{n} \boldsymbol{u}_{h}^{n}, \frac{\boldsymbol{v}_{h}^{n+1}-\boldsymbol{v}_{h}^{n}}{k}\right)-\left(\rho_{0 h} \boldsymbol{u}_{0 h}, \boldsymbol{v}_{h}^{0}\right) \\
+k \sum_{n=0}^{N-1} c\left(\rho_{h}^{n+1} \boldsymbol{u}_{h}^{n}-\lambda \nabla \rho_{h}^{n+1}, \boldsymbol{u}_{h}^{n+1}, \boldsymbol{v}_{h}^{n+1}\right)+k \sum_{n=0}^{N-1} a\left(\boldsymbol{u}_{h}^{n+1}, \boldsymbol{v}_{h}^{n+1}\right) \\
=k \sum_{n=0}^{N-1}\left(\rho_{h}^{n+1} \boldsymbol{f}^{n+1}, \boldsymbol{v}_{h}^{n+1}\right)+k \sum_{n=0}^{N-1} \frac{1}{2}\left(\frac{\rho_{h}^{n+1}-\rho_{h}^{n}}{k}, \boldsymbol{u}_{h}^{n+1} \cdot \boldsymbol{v}_{h}^{n+1}\right) .
\end{array}\right.
$$

Next, by taking into account Definition 5.1,

$$
\left\{\begin{array}{l}
-\int_{0}^{T}\left(\widehat{\rho}_{h, k} \widehat{\boldsymbol{u}}_{h, k}, \frac{\partial}{\partial t} \widetilde{\boldsymbol{v}}_{h, k}\right)-\left(\rho_{0 h} \boldsymbol{u}_{0 h}, \boldsymbol{v}_{h}^{0}\right) \\
+\int_{0}^{T} c\left(\rho_{h, k} \widehat{\boldsymbol{u}}_{h, k}-\lambda \nabla \rho_{h, k}, \boldsymbol{u}_{h, k}, \boldsymbol{v}_{h, k}\right)+\sum_{n=0}^{N-1} a\left(\rho_{h, k}, \boldsymbol{u}_{h, k}, \boldsymbol{v}_{h, k}\right) \\
=\int_{0}^{T}\left(\rho_{h, k} \boldsymbol{f}_{k}, \boldsymbol{v}_{h, k}\right)+\frac{1}{2} \int_{0}^{T}\left(\frac{\partial}{\partial t} \widetilde{\rho}_{h, k}, \boldsymbol{u}_{h, k} \cdot \boldsymbol{v}_{h, k}\right) .
\end{array}\right.
$$

This variational formulation of the discrete momentum system allows us to pass to the limit in a standard way. We pass to the limit only in the last term on the righthand side since this term does not appear in the theoretical analysis. We know that $\frac{\partial}{\partial t} \widetilde{\rho}_{h, k} \rightarrow \frac{\partial}{\partial t} \rho$ weakly in $L^{4 / 3}\left(0, T ; L^{2}(\Omega)\right)$ and $\boldsymbol{u}_{h, k} \rightarrow \boldsymbol{u}$ strongly in $L^{p}\left(0, T ; L^{2}(\Omega)\right)$, with $p<\infty$, and is bounded in $L^{4}\left(0, T ; \boldsymbol{L}^{3}(\Omega)\right)$, and hence $\frac{\partial}{\partial t} \widetilde{\rho}_{h, k} \boldsymbol{u}_{h, k} \rightarrow \frac{\partial}{\partial t} \rho \boldsymbol{u}$ weakly in $L^{1}\left(0, T ; \boldsymbol{L}^{6 / 5}(\Omega)\right)$. As $\boldsymbol{v}_{h, k} \rightarrow \boldsymbol{v}$ in $L^{\infty}\left(0, T ; \boldsymbol{H}_{0}^{1}(\Omega)\right)$, we have

$$
\int_{0}^{T}\left(\frac{\partial}{\partial t} \widetilde{\rho}_{h, k}, \boldsymbol{u}_{h, k} \cdot \boldsymbol{v}_{h, k}\right) \rightarrow \int_{0}^{T}\left(\frac{\partial}{\partial t} \rho, \boldsymbol{u} \cdot \boldsymbol{v}\right) \text { as }(h, k) \rightarrow 0 .
$$

This concludes the proof of Theorem 1.1.

Remark 7.2. A variant of the Kazhikhoz-Smagulov model is obtained by replacing the linear diffusion term $-\nabla \cdot(\mu \nabla \boldsymbol{u})$ in (1.1) by a nonlinear diffusion term $-\lambda \nabla \cdot(\rho \nabla \boldsymbol{u})$ (i.e., taking $\mu=\lambda \rho$ ). It is a model of pollution studied by Bresch, Essoufi, and Sy in $[4,5]$, where they prove the existence of a global in time weak solution, without imposing the restrictive hypothesis (1.5) on the coefficients.

The scheme that we design for this model is obtained by replacing the stabilizing term of the momentum system

$$
-\lambda \int_{\Omega} \frac{M+m}{2}\left(\nabla \boldsymbol{u}_{h}^{n+1}\right)^{t}: \nabla \overline{\boldsymbol{u}}_{h} d \boldsymbol{x}
$$

by the term $\frac{\lambda m}{2}\left(\nabla \cdot \boldsymbol{u}_{h}^{n+1}, \nabla \cdot \overline{\boldsymbol{u}}_{h}\right)$ and the remainder with the following scheme:

Given $\left(\rho_{h}^{n}, \boldsymbol{u}_{h}^{n}, p_{h}^{n}\right) \in W_{h} \times \boldsymbol{V}_{h} \times M_{h}$, 
1. find $\left(\boldsymbol{w}_{h}^{n}, q_{h}^{n}\right) \in \widetilde{\boldsymbol{V}}_{h} \times \widetilde{M}_{h}$ such that, for each $\left(\overline{\boldsymbol{w}}_{h}, \bar{q}_{h}\right) \in \widetilde{\boldsymbol{V}}_{h} \times \widetilde{M}_{h}$,

$$
\left(\nabla \boldsymbol{w}_{h}^{n}, \nabla \overline{\boldsymbol{w}}_{h}\right)-\left(q_{h}^{n}, \nabla \cdot \overline{\boldsymbol{w}}_{h}\right)=\left(\nabla \boldsymbol{u}_{h}^{n}, \nabla \overline{\boldsymbol{w}}_{h}\right), \quad\left(\nabla \cdot \boldsymbol{w}_{h}^{n}, \bar{q}_{h}\right)=0
$$

2. find $\rho_{h}^{n+1} \in W_{h}$ such that, for each $\bar{\rho}_{h} \in W_{h}$,

$$
\left(\frac{\rho_{h}^{n+1}-\rho_{h}^{n}}{k}, \bar{\rho}_{h}\right)+\left(\boldsymbol{w}_{h}^{n} \cdot \nabla \rho_{h}^{n+1}, \bar{\rho}_{h}\right)+\lambda\left(\nabla \rho_{h}^{n+1}, \nabla \bar{\rho}_{h}\right)=0
$$

3. find $\left(\boldsymbol{u}_{h}^{n+1}, p_{h}^{n+1}\right) \in \boldsymbol{V}_{h} \times M_{h}$ such that, for each $\left(\overline{\boldsymbol{u}}_{h}, \overline{p_{h}}\right) \in \boldsymbol{V}_{h} \times M_{h}$,

$$
\left\{\begin{array}{c}
\left(\rho_{h}^{n} \frac{\boldsymbol{u}_{h}^{n+1}-\boldsymbol{u}_{h}^{n}}{k}, \overline{\boldsymbol{u}}_{h}\right)+\frac{1}{2}\left(\frac{\rho_{h}^{n+1}-\rho_{h}^{n}}{k}, \boldsymbol{u}_{h}^{n+1} \cdot \overline{\boldsymbol{u}}_{h}\right) \\
+c\left(\rho_{h}^{n+1} \boldsymbol{u}_{h}^{n}-\lambda \nabla \rho_{h}^{n+1}, \boldsymbol{u}_{h}^{n+1}, \overline{\boldsymbol{u}}_{h}\right) \\
+\lambda\left(\rho_{h}^{n+1}\left(\nabla \boldsymbol{u}_{h}^{n+1}-\left(\nabla \boldsymbol{u}_{h}^{n+1}\right)^{t}\right), \nabla \overline{\boldsymbol{u}}_{h}\right)+\frac{\lambda m}{2}\left(\nabla \cdot \boldsymbol{u}_{h}^{n+1}, \nabla \cdot \overline{\boldsymbol{u}}_{h}\right) \\
=\left(\rho_{h}^{n+1} \boldsymbol{f}^{n+1}, \overline{\boldsymbol{u}}_{h}\right)+\left(p_{h}^{n+1}, \nabla \cdot \overline{\boldsymbol{u}}_{h}\right), \\
\left(\nabla \cdot \boldsymbol{u}_{h}^{n+1}, \bar{p}_{h}\right)=0 .
\end{array}\right.
$$

By following the arguments of this paper, one may establish the same conclusions of Theorem 1.1 for this scheme.

8. Asymptotic behavior when $\boldsymbol{\lambda} \rightarrow \mathbf{0}$. In this section we are interested in the asymptotic behavior of scheme (1.8)-(1.10) when the diffusion parameter $\lambda$ goes to zero. More precisely, we will see that, by imposing the stability condition

$$
h /\left(k \lambda^{2}\right) \rightarrow 0 \quad \text { as }(\lambda, h, k) \rightarrow 0
$$

and completing (H2) with the additional approximation property

$$
\left|\bar{\rho}-I_{h} \bar{\rho}\right| \leq C h^{2 / 3}\|\bar{\rho}\|_{W^{1,3 / 2}(\Omega)} \quad \forall \bar{\rho} \in W^{1,3 / 2}(\Omega),
$$

then scheme (1.8)-(1.10) approximates, as $(h, k, \lambda) \rightarrow 0$, to a weak solution of the density-dependent Navier-Stokes problem (1.6), which is defined as follows.

Definition 8.1. A pair $(\rho, \boldsymbol{u})$ is said to be a weak solution of $(1.6)$ in $(0, T)$ if:

(a) $\boldsymbol{u} \in L^{\infty}\left(0, T ; \boldsymbol{L}^{2}(\Omega)\right) \cap L^{2}(0, T ; \boldsymbol{V}), \rho \in L^{\infty}(Q)$, with $0<m \leq \rho(\boldsymbol{x}, t) \leq M$ a.e. $(\boldsymbol{x}, t) \in Q$;

(b) for all $\phi \in C^{1}([0, T] ; \boldsymbol{V})$, with $\phi(T)=0$,

$$
\int_{0}^{T}\left\{-\left(\rho \boldsymbol{u}, \phi_{t}+(\boldsymbol{u} \cdot \nabla) \phi\right)+\mu(\nabla \boldsymbol{u}, \nabla \phi)\right\} d t=\int_{0}^{T}(\rho \boldsymbol{f}, \phi) d t+\left(\rho_{0} \boldsymbol{u}_{0}, \phi(0)\right)
$$

(c) for all $\varphi \in C^{1}\left([0, T] ; \boldsymbol{H}^{1}(\Omega)\right)$, with $\varphi(T)=0$,

$$
-\int_{0}^{T}\left(\rho, \varphi_{t}\right) d t-\int_{0}^{T}(\rho \boldsymbol{u}, \nabla \varphi) d t=\left(\rho_{0}, \varphi(0)\right) .
$$

The rest of this section is devoted to the proof of Theorem 1.2.

Copyright $@$ by SIAM. Unauthorized reproduction of this article is prohibited. 
8.1. Uniform estimates with respect to $(h, k, \lambda)$. By following arguments of the previous sections and assuming $\left(\mathrm{S}^{\prime}\right)$ and $(h, k, \lambda)$ small enough, we can obtain the following estimates independent of $h, k$, and $\lambda$ (now we denote piecewise functions associated to the scheme also with the parameter $\lambda$ explicitly):

$$
\begin{gathered}
\left\{\boldsymbol{u}_{h, k, \lambda}\right\}_{h, k, \lambda},\left\{\widehat{\boldsymbol{u}}_{h, k, \lambda}\right\}_{h, k, \lambda},\left\{\widehat{\boldsymbol{w}}_{h, k, \lambda}\right\}_{h, k, \lambda} \text { in } L^{\infty}\left(0, T ; \boldsymbol{L}^{2}(\Omega)\right) \cap L^{2}\left(0, T ; \boldsymbol{H}_{0}^{1}(\Omega)\right), \\
\left\{\widetilde{\rho}_{h, k, \lambda}\right\}_{h, k, \lambda},\left\{\rho_{h, k, \lambda}\right\}_{h, k, \lambda},\left\{\widehat{\rho}_{h, k, \lambda}\right\}_{h, k, \lambda} \text { in } L^{\infty}(Q), \\
\lambda^{1 / 2}\left\{\rho_{h, k, \lambda}\right\}_{h, k, \lambda}, \lambda^{1 / 2}\left\{\widehat{\rho}_{h, k, \lambda}\right\}_{h, k, \lambda} \text { in } L^{\infty}\left(0, T ; H^{1}(\Omega)\right), \\
\lambda^{3 / 4}\left\{\rho_{h, k, \lambda}\right\}_{h, k, \lambda} \text { in } L^{4}\left(0, T ; W^{1,3}(\Omega)\right), \\
\lambda^{3 / 4}\left\{\frac{\partial}{\partial t} \widetilde{\rho}_{h, k, \lambda}\right\}_{h, k, \lambda} \text { in } L^{4 / 3}\left(0, T ; L^{2}(\Omega)\right) .
\end{gathered}
$$

In addition, $0<\widetilde{m} \leq \rho_{h, k, \lambda}, \widehat{\rho}_{h, k, \lambda}, \widetilde{\rho}_{h, k, \lambda} \leq \widetilde{M}$ in $Q$,

$$
\begin{aligned}
\left\|\widetilde{\rho}_{h, k, \lambda}-\rho_{h, k, \lambda}\right\|_{L^{2}\left(0, T ; L^{2}(\Omega)\right)} & \leq\left\|\rho_{h, k, \lambda}-\widehat{\rho}_{h, k, \lambda}\right\|_{L^{2}\left(0, T ; L^{2}(\Omega)\right)} \leq C \sqrt{k}, \\
\left\|\boldsymbol{u}_{h, k, \lambda}-\widehat{\boldsymbol{u}}_{h, k, \lambda}\right\|_{L^{2}\left(0, T ; \boldsymbol{H}_{0}^{1}(\Omega)\right)} & \leq C \sqrt{k} .
\end{aligned}
$$

In fact, we have the following result.

LEMma 8.2. By assuming $\left(\mathrm{S}^{\prime}\right)$ and $(h, k, \lambda)$ small enough, there exist subsequences of $\left\{\boldsymbol{u}_{h, k, \lambda}\right\}_{h, k},\left\{\widehat{\boldsymbol{u}}_{h, k, \lambda}\right\}_{h, k},\left\{\rho_{h, k, \lambda}\right\}_{h, k, \lambda},\left\{\widehat{\rho}_{h, k, \lambda}\right\}_{h, k}$, and $\left\{\widetilde{\rho}_{h, k, \lambda}\right\}_{h, k}$ (denoted in the same way) and limit functions $\boldsymbol{u}$ and $\rho$, such that $\nabla \cdot \boldsymbol{u}=0$ a.e. in $\Omega$, and the following weak convergences hold as $(h, k, \lambda) \rightarrow 0$ :

$$
\begin{gathered}
\boldsymbol{u}_{h, k, \lambda} \rightarrow \boldsymbol{u}, \quad \widehat{\boldsymbol{u}}_{h, k, \lambda} \rightarrow \boldsymbol{u}, \quad \widehat{\boldsymbol{w}}_{h, k, \lambda} \rightarrow \boldsymbol{u} \quad \text { in }\left\{\begin{array}{l}
L^{2}\left(0, T ; \boldsymbol{H}_{0}^{1}(\Omega)\right) \text {-weak }, \\
L^{\infty}\left(0, T ; \boldsymbol{L}^{2}(\Omega)\right) \text {-weak },
\end{array}\right. \\
\widetilde{\rho}_{h, k, \lambda} \rightarrow \rho, \quad \rho_{h, k, \lambda} \rightarrow \rho, \quad \widehat{\rho}_{h, k, \lambda} \rightarrow \rho \quad \text { in } L^{\infty}(Q) \text {-weak } * .
\end{gathered}
$$

\subsection{Compactness.}

Proposition 8.3. Under the hypotheses of Lemma 8.2, the estimate

$$
\int_{0}^{T-\delta}\left|\sqrt{\rho_{h, k, \lambda}(t+\delta)}\left(\boldsymbol{u}_{h, k, \lambda}(t+\delta)-\boldsymbol{u}_{h, k, \lambda}(t)\right)\right|^{2} d t \leq C \delta^{1 / 2} \quad \forall \delta: 0<\delta<T
$$

holds, with $C>0$ independent of $h, k, \delta$, and $\lambda$.

In particular, since $\rho_{h, k, \lambda} \geq \widetilde{m}$, then

$$
\boldsymbol{u}_{h, k, \lambda} \rightarrow \boldsymbol{u}, \quad \boldsymbol{w}_{h, k, \lambda} \rightarrow \boldsymbol{u} \quad \text { in } L^{2}\left(0, T ; \boldsymbol{L}^{2}(\Omega)\right) \text { as }(h, k, \lambda) \rightarrow 0 .
$$

Proof. Again, since $\rho_{h, k, \lambda}$ and $\boldsymbol{u}_{h, k, \lambda}$ are piecewise constant functions, (8.1) is equivalent to

$$
k \sum_{m=0}^{N-r}\left|\sqrt{\rho_{h}^{m+r}}\left(\boldsymbol{u}_{h}^{m+r}-\boldsymbol{u}_{h}^{m}\right)\right|^{2} \leq C(r k)^{1 / 2} \quad \forall r: 0 \leq r \leq N .
$$

By following the proof of Proposition 6.3, one observes that the terms which are not necessarily bounded independent of $\lambda$ are

$$
T_{1}:=k^{2} \sum_{m=0}^{N-r} \sum_{n=m}^{m-1+r}\left(\frac{\rho_{h}^{n+1}-\rho_{h}^{n}}{k}, \boldsymbol{u}_{h}^{n+1} \cdot\left(\boldsymbol{u}_{h}^{m+r}-\boldsymbol{u}_{h}^{m}\right)\right)
$$

Copyright $@$ by SIAM. Unauthorized reproduction of this article is prohibited. 
and

$$
T_{2}:=-k \sum_{m=0}^{N-r}\left(\rho_{h}^{m+r}-\rho_{h}^{m}, \boldsymbol{u}_{h}^{m} \cdot\left(\boldsymbol{u}_{h}^{m+r}-\boldsymbol{u}_{h}^{m}\right)\right)
$$

Here we are going to bound these terms.

We consider the projection operator on $W_{h}$ with respect to $L^{2}(\Omega)$-inner product:

$$
P_{h}: L^{2}(\Omega) \rightarrow W_{h} \quad \text { such that } \quad\left(P_{h} v, w\right)=(v, w) \quad \forall w \in W_{h} .
$$

We take $\bar{\rho}_{h}=P_{h}\left(\boldsymbol{u}_{h}^{n+1} \cdot\left(\boldsymbol{u}_{h}^{m+r}-\boldsymbol{u}_{h}^{m}\right)\right) \in W_{h}$ in (4.2), arriving at

$$
\begin{aligned}
& \left(\frac{\rho_{h}^{n+1}-\rho_{h}^{n}}{k}, \boldsymbol{u}_{h}^{n+1} \cdot\left(\boldsymbol{u}_{h}^{m+r}-\boldsymbol{u}_{h}^{m}\right)\right)+\left(\boldsymbol{w}_{h}^{n} \cdot \nabla \rho_{h}^{n+1}, P_{h}\left(\boldsymbol{u}_{h}^{n+1} \cdot\left(\boldsymbol{u}_{h}^{m+r}-\boldsymbol{u}_{h}^{m}\right)\right)\right) \\
& \quad-\lambda\left(\Delta_{h} \rho_{h}^{n+1}, \boldsymbol{u}_{h}^{n+1} \cdot\left(\boldsymbol{u}_{h}^{m+r}-\boldsymbol{u}_{h}^{m}\right)\right)=0
\end{aligned}
$$

where we have used the definition of projection operator in $L^{2}(\Omega)$ in the first and the last term. Thus, we decompose $T_{1}=T_{1,1}+T_{1,2}$, where:

$$
\begin{gathered}
T_{1,1}=-k^{2} \sum_{m=0}^{N-r} \sum_{n=m}^{m-1+r}\left(\boldsymbol{w}_{h}^{n} \cdot \nabla \rho_{h}^{n+1}, P_{h}\left(\boldsymbol{u}_{h}^{n+1} \cdot\left(\boldsymbol{u}_{h}^{m+r}-\boldsymbol{u}_{h}^{m}\right)\right)\right), \\
T_{1,2}=\lambda k^{2} \sum_{m=0}^{N-r} \sum_{n=m}^{m-1+r}\left(\Delta_{h} \rho_{h}^{n+1}, \boldsymbol{u}_{h}^{n+1} \cdot\left(\boldsymbol{u}_{h}^{m+r}-\boldsymbol{u}_{h}^{m}\right)\right) .
\end{gathered}
$$

By writing $T_{1,1}$ as follows:

$$
\begin{aligned}
T_{1,1} & =-k^{2} \sum_{m=0}^{N-r} \sum_{n=m}^{m-1+r}\left(\boldsymbol{w}_{h}^{n} \cdot \nabla \rho_{h}^{n+1}, P_{h}\left(\boldsymbol{u}_{h}^{n+1} \cdot\left(\boldsymbol{u}_{h}^{m+r}-\boldsymbol{u}_{h}^{m}\right)\right)-\boldsymbol{u}_{h}^{n+1} \cdot\left(\boldsymbol{u}_{h}^{m+r}-\boldsymbol{u}_{h}^{m}\right)\right) \\
& -k^{2} \sum_{m=0}^{N-r} \sum_{n=m}^{m-1+r}\left(\boldsymbol{w}_{h}^{n} \cdot \nabla \rho_{h}^{n+1}, \boldsymbol{u}_{h}^{n+1} \cdot\left(\boldsymbol{u}_{h}^{m+r}-\boldsymbol{u}_{h}^{m}\right)\right):=T_{1,1}^{1}+T_{1,1}^{2},
\end{aligned}
$$

we bound it as

$$
T_{1,1}^{1} \leq C k^{2} \sum_{m=0}^{N-r} \sum_{n=m}^{m-1+r}\left\|\boldsymbol{w}_{h}^{n}\right\|_{L^{6}(\Omega)}\left\|\nabla \rho_{h}^{n+1}\right\|_{L^{3}(\Omega)} h^{2 / 3}\left\|\boldsymbol{u}_{h}^{n+1}\right\|\left\|\boldsymbol{u}_{h}^{m+r}-\boldsymbol{u}_{h}^{m}\right\|,
$$

where we have used the inequalities $\left|\bar{\rho}-P_{h} \bar{\rho}\right| \leq\left|\bar{\rho}-I_{h} \bar{\rho}\right| \leq C h^{2 / 3}\|\bar{\rho}\|_{W^{1,3 / 2}(\Omega)}$ and $\left\|\boldsymbol{u}_{h}^{n+1} \cdot\left(\boldsymbol{u}_{h}^{m+r}-\boldsymbol{u}_{h}^{m}\right)\right\|_{W^{1,3 / 2}(\Omega)} \leq\left\|\boldsymbol{u}_{h}^{n+1}\right\|\left\|\boldsymbol{u}_{h}^{m+r}-\boldsymbol{u}_{h}^{m}\right\|$. Next, by taking $(\lambda, h, k)$ small enough and such that

$$
h^{2 / 3} \leq C \lambda^{3 / 4} k^{1 / 2},
$$

using the fact that $\lambda^{3 / 4} k^{1 / 2}\left\|\nabla \rho_{h}^{n+1}\right\|_{L^{3}(\Omega)} \leq C$ (with $C$ independent of $\lambda, h, k$ ), and applying the discrete Fubini rule, we arrive at the estimate $T_{1,1}^{1} \leq C(r k)^{1 / 2}$.

Copyright $@$ by SIAM. Unauthorized reproduction of this article is prohibited. 
The bound $T_{1,1}^{2} \leq C(r k)^{1 / 2}$ is obtained easily by integrating by parts and using the pointwise bound $\left\|\rho_{h}^{n+1}\right\|_{L^{\infty}(\Omega)} \leq C$ (where $C$ is independent of $\lambda$ ):

$$
\begin{aligned}
T_{1,1}^{2} & \leq k^{2} \sum_{m=0}^{N-r} \sum_{n=m}^{m-1+r}\left(\left|\nabla \cdot \boldsymbol{w}_{h}^{n}\right|\left|\boldsymbol{u}_{h}^{n+1} \cdot\left(\boldsymbol{u}_{h}^{m+r}-\boldsymbol{u}_{h}^{m}\right)\right|+\left\|\boldsymbol{w}_{h}^{n}\right\|_{L^{6}}\left|\nabla \boldsymbol{u}_{h}^{n+1}\right|\left\|\boldsymbol{u}_{h}^{m+r}-\boldsymbol{u}_{h}^{m}\right\|_{L^{3}}\right) \\
& +k^{2} \sum_{m=0}^{N-r} \sum_{n=m}^{m-1+r}\left\|\boldsymbol{w}_{h}^{n}\right\|_{L^{6}}\left\|\boldsymbol{u}_{h}^{n+1}\right\|_{L^{3}}\left|\nabla\left(\boldsymbol{u}_{h}^{m+r}-\boldsymbol{u}_{h}^{m}\right)\right| \\
& \leq C k^{2} \sum_{n=0}^{N-1}\left\|\boldsymbol{w}_{h}^{n}\right\|\left\|\boldsymbol{u}_{h}^{n+1}\right\| \sum_{m=\overline{n-r+1}}^{\bar{n}}\left\|\boldsymbol{u}_{h}^{m+r}-\boldsymbol{u}^{m}\right\| \leq C(r k)^{1 / 2}
\end{aligned}
$$

The bound $T_{1,2} \leq C(r k)^{1 / 2}$ is obtained using the fact that $\lambda^{2} k \sum_{n=0}^{N-1}\left|\Delta_{h} \rho_{h}^{n+1}\right|^{2} \leq C$ and Fubini's rule.

By summing up (8.3) multiplied by $k^{2}$ for $n=m, \ldots, m+r-1$ and then summing up for $m=0, \ldots, N-r$, we can write $T_{2}$ as:

$T_{2}=-k^{2} \sum_{m=0}^{N-r} \sum_{n=m}^{m+r-1}\left(\boldsymbol{w}_{h}^{n} \cdot \nabla \rho_{h}^{n}, P_{h}\left(\boldsymbol{u}_{h}^{m} \cdot\left(\boldsymbol{u}_{h}^{m+r}-\boldsymbol{u}_{h}^{m}\right)\right)-\lambda\left(\Delta_{h} \rho_{h}^{n+1}, \boldsymbol{u}_{h}^{m} \cdot\left(\boldsymbol{u}_{h}^{m+r}-\boldsymbol{u}_{h}^{m}\right)\right)\right.$.

These new terms are bounded in an analogous manner (now, without using Fubinis's rule), resulting in

$$
T_{2} \leq C(r k)^{1 / 2} .
$$

Note that the restriction on the parameters (8.4) imposed to get the bound of $T_{1,1}^{1}$ is included in the constraint $\left(\mathrm{S}^{\prime}\right)$ imposed to obtain a priori estimates. Indeed, (8.4) is equivalent to $\frac{1}{\lambda^{3 / 4}} \frac{h^{2 / 3}}{k^{1 / 4}} \leq C$. But as

$$
\lim _{(h, k, \lambda) \rightarrow 0}\left(\frac{1}{\lambda} \sqrt{\frac{h}{k}}\right) /\left(\frac{1}{\lambda^{3 / 4}} \frac{h^{2 / 3}}{k^{1 / 4}}\right)=\lim _{(h, k, \lambda) \rightarrow 0} \frac{1}{\lambda^{1 / 4} k^{1 / 2} h^{1 / 6}}=\infty,
$$

then $\frac{1}{\lambda^{3 / 4}} \frac{h^{2 / 3}}{k^{1 / 4}} \leq C \frac{1}{\lambda} \sqrt{\frac{h}{k}} \rightarrow 0$ (thanks to $\left(\mathrm{S}^{\prime}\right)$ ).

Remark 8.4. By comparing the fractional in time estimates for the discrete velocity in the case of $\lambda$ fixed (done in Proposition 6.3) with respect to the case of $\lambda \rightarrow 0$ (done now in Proposition 8.3), one can observe that condition (8.4) on the parameters $h$ and $k$ imposed now in the proof of Proposition 8.3 is not necessary in the case of $\lambda$ fixed; however, in this case only order $(r k)^{1 / 4}$ is obtained. Now, in the case $\lambda \rightarrow 0$, we have to impose constraint (8.4), and the estimate order is improved from $(r k)^{1 / 4}$ to $(r k)^{1 / 2}$.

\subsection{Passing to the limit.}

8.3.1. Density equation. Let $\eta \in C^{1}\left([0, T] ; C_{c}^{\infty}(\Omega)\right)$ such that $\eta(T)=0$. We define $\eta_{h}^{n}$ as the interpolation in $W_{h}$ of $\eta\left(t_{n}\right)$ and $\left.\eta_{h, k} \in L^{\infty}\left(0, T ; W_{h}\right)\right)$ as the piecewise constant function taking values $\eta_{h}^{n+1}$ in $\left(t_{n}, t_{n+1}\right]$ and let $\left.\widetilde{\eta}_{h, k} \in C^{0}\left([0, T] ; W_{h}\right)\right)$ be the piecewise linear, globally continuous function such that $\widetilde{\eta}_{h, k}\left(t_{n}\right)=\eta_{h}^{n}$. One has, as $(h, k) \rightarrow 0$,

$$
\eta_{k, h} \rightarrow \eta \quad \text { in } L^{\infty}\left(0, T ; H^{1}(\Omega)\right) \quad \text { and } \quad \widetilde{\eta}_{k, h} \rightarrow \eta \quad \text { in } W^{1, \infty}\left(0, T ; H^{1}(\Omega)\right) .
$$


By using these discrete test functions in the discrete density formulation (1.8), together with an integration by parts in time, we arrive [11] at the formulation

$$
\int_{0}^{T}\left(\widehat{\rho}_{h, k, \lambda},-\frac{d}{d t} \widetilde{\eta}_{h, k}\right)+\lambda\left(\nabla \rho_{h, k, \lambda}, \nabla \eta_{h, k}\right)+\left(\widehat{\boldsymbol{w}}_{h, k, \lambda} \cdot \nabla \rho_{h, k, \lambda}, \eta_{h, k}\right)=\left(\rho_{0 h}, \eta_{h}^{0}\right) .
$$

Before taking a limit in (8.5), we rewrite the convective term in the following form:

$$
\begin{gathered}
\int_{0}^{T}\left(\widehat{\boldsymbol{w}}_{h, k, \lambda} \cdot \nabla \rho_{h, k, \lambda}, \eta_{h, k}\right) d t=-\int_{0}^{T}\left(\nabla \cdot \widehat{\boldsymbol{w}}_{h, k, \lambda} \rho_{h, k, \lambda}, \eta_{h, k}\right) d t \\
-\int_{0}^{T}\left(\rho_{h, k, \lambda} \widehat{\boldsymbol{w}}_{h, k, \lambda}, \nabla \eta_{h, k}\right) d t=-\int_{0}^{T}\left(\rho_{h, k, \lambda} \widehat{\boldsymbol{w}}_{h, k, \lambda}, \nabla \eta_{h, k}\right) d t
\end{gathered}
$$

where we have used Remark 3.1. Therefore, (8.5) remains as

$$
\int_{0}^{T}\left(\widehat{\rho}_{h, k, \lambda},-\frac{d}{d t} \widetilde{\eta}_{h, k}\right)+\lambda\left(\nabla \rho_{h, k, \lambda}, \nabla \eta_{h, k}\right)-\left(\widehat{\boldsymbol{w}}_{h, k, \lambda} \rho_{h, k, \lambda}, \nabla \eta_{h, k}\right)=\left(\rho_{0 h}, \eta_{h}^{0}\right) .
$$

By taking into account the weak and strong convergences obtained in previous subsections, it is possible to pass to the limit. Notice that, by arguing as in [9], one can prove that

$$
\lambda \int_{0}^{T}\left(\nabla \rho_{h, k, \lambda}, \nabla \eta_{h, k}\right) d t \rightarrow 0 \quad \text { as }(h, k, \lambda) \rightarrow 0
$$

8.3.2. Velocity system. Before taking a limit in the discrete momentum system (1.9), we will write (1.9) in a completely conservative form. For this, summing to both sides of (1.9) the terms

$$
\frac{1}{2}\left(\frac{\rho_{h}^{n+1}-\rho_{h}^{n}}{k}, \boldsymbol{u}_{h}^{n+1} \cdot \overline{\boldsymbol{u}}_{h}\right)-\frac{1}{2}\left(\rho_{h}^{n+1} \boldsymbol{u}_{h}^{n}-\lambda \nabla \rho_{h}^{n+1}, \nabla\left(\boldsymbol{u}_{h}^{n+1} \cdot \overline{\boldsymbol{u}}_{h}\right)\right)
$$

provides that, for each $\overline{\boldsymbol{u}}_{h} \in \mathbf{V}_{h}$,

$$
\left\{\begin{array}{l}
\left(\frac{\rho_{h}^{n+1} \boldsymbol{u}_{h}^{n+1}-\rho_{h}^{n} \boldsymbol{u}_{h}^{n}}{k}, \overline{\boldsymbol{u}}_{h}\right)-\left(\left(\rho_{h}^{n+1} \boldsymbol{u}_{h}^{n}-\lambda \nabla \rho_{h}^{n+1}\right) \otimes \boldsymbol{u}_{h}^{n+1}, \nabla \overline{\boldsymbol{u}}_{h}\right) \\
+a\left(\rho_{h}^{n+1}, \boldsymbol{u}_{h}^{n+1}, \overline{\boldsymbol{u}}_{h}\right)=\left(\rho_{h}^{n+1} \boldsymbol{f}^{n+1}, \overline{\boldsymbol{u}}_{h}\right)+\left(p_{h}^{n+1}, \nabla \cdot \overline{\boldsymbol{u}}_{h}\right) \\
+\frac{1}{2}\left(\frac{\rho_{h}^{n+1}-\rho_{h}^{n}}{k}, \boldsymbol{u}_{h}^{n+1} \cdot \overline{\boldsymbol{u}}_{h}\right)-\frac{1}{2}\left(\rho_{h}^{n+1} \boldsymbol{u}_{h}^{n}-\lambda \nabla \rho_{h}^{n+1}, \nabla\left(\boldsymbol{u}_{h}^{n+1} \cdot \overline{\boldsymbol{u}}_{h}\right)\right)
\end{array}\right.
$$

Now we consider $Q_{h}$ the projector operator onto $W_{h}$ with respect to the $H^{1}$-norm:

$$
Q_{h}: H^{1}(\Omega) \rightarrow W_{h} \text { such that }\left(Q_{h} v, w\right)_{H^{1}(\Omega)}=(v, w)_{H^{1}(\Omega)} \quad \forall w \in W_{h},
$$

where $(\cdot, \cdot)_{H^{1}(\Omega)}$ denotes the usual $H^{1}$-inner product.

Copyright (c) by SIAM. Unauthorized reproduction of this article is prohibited. 
By subtracting from the second member of (8.6) the result of taking $\bar{\rho}_{h}=\frac{1}{2} Q_{h}\left(\boldsymbol{u}_{h}^{n+1}\right.$. $\left.\bar{u}_{h}\right)$ as a test function in (1.8) and integrating by parts the convective term, one has

$$
\left\{\begin{array}{l}
\left(\frac{\rho_{h}^{n+1} \boldsymbol{u}_{h}^{n+1}-\rho_{h}^{n} \boldsymbol{u}_{h}^{n}}{k}, \overline{\boldsymbol{u}}_{h}\right)-\left(\left(\rho_{h}^{n+1} \boldsymbol{u}_{h}^{n}-\lambda \nabla \rho_{h}^{n+1}\right) \otimes \boldsymbol{u}_{h}^{n+1}, \nabla \overline{\boldsymbol{u}}_{h}\right) \\
+a\left(\rho_{h}^{n+1}, \boldsymbol{u}_{h}^{n+1}, \overline{\boldsymbol{u}}_{h}\right)=\left(\rho_{h}^{n+1} \boldsymbol{f}^{n+1}, \overline{\boldsymbol{u}}_{h}\right)+\left(p_{h}^{n+1}, \nabla \cdot \overline{\boldsymbol{u}}_{h}\right) \\
+\frac{1}{2}\left(\frac{\rho_{h}^{n+1}-\rho_{h}^{n}}{k}, \boldsymbol{u}_{h}^{n+1} \cdot \overline{\boldsymbol{u}}_{h}-Q_{h}\left(\boldsymbol{u}_{h}^{n+1} \cdot \overline{\boldsymbol{u}}_{h}\right)\right) \\
-\frac{1}{2}\left(\rho_{h}^{n+1} \boldsymbol{w}_{h}^{n}, \nabla\left(\boldsymbol{u}_{h}^{n+1} \cdot \overline{\boldsymbol{u}}_{h}-Q_{h}\left(\boldsymbol{u}_{h}^{n+1} \cdot \overline{\boldsymbol{u}}_{h}\right)\right)\right) \\
-\frac{1}{2}\left(\rho_{h}^{n+1}\left(\boldsymbol{u}_{h}^{n}-\boldsymbol{w}_{h}^{n}\right), \nabla\left(\boldsymbol{u}_{h}^{n+1} \cdot \overline{\boldsymbol{u}}_{h}\right)\right)+\frac{1}{2}\left(\nabla \cdot \boldsymbol{w}_{h}^{n} \rho_{h}^{n+1}, Q_{h}\left(\boldsymbol{u}_{h}^{n+1} \cdot \overline{\boldsymbol{u}}_{h}\right)\right) \\
-\frac{\lambda}{2}\left(\rho_{h}^{n+1}, \boldsymbol{u}_{h}^{n+1} \cdot \overline{\boldsymbol{u}}_{h}-Q_{h}\left(\boldsymbol{u}_{h}^{n+1} \cdot \overline{\boldsymbol{u}}_{h}\right)\right),
\end{array}\right.
$$

where we have used the definition of $Q_{h}$ in the last term on the right-hand side. Note that the term $\left(\nabla \cdot \boldsymbol{w}_{h}^{n} \rho_{h}^{n+1}, Q_{h}\left(\boldsymbol{u}_{h}^{n+1} \cdot \overline{\boldsymbol{u}}_{h}\right)\right)=0$ thanks to Remark 3.1.

In a similar way to section 7 , we arrive at

$$
\left\{\begin{array}{l}
-\int_{0}^{T}\left(\widehat{\rho}_{h, k, \lambda} \widehat{\boldsymbol{u}}_{h, k, \lambda}, \frac{\partial}{\partial t} \widetilde{\boldsymbol{v}}_{h, k}\right)-\left(\rho_{0 h} \boldsymbol{u}_{0 h}, \boldsymbol{v}_{h}^{0}\right) \\
-\int_{0}^{T}\left(\rho_{h, k, \lambda} \widehat{\boldsymbol{u}}_{h, k}-\lambda \nabla \rho_{h, k, \lambda} \otimes \boldsymbol{u}_{h, k, \lambda}, \nabla \boldsymbol{v}_{h, k}\right)+a\left(\rho_{h, k, \lambda}, \boldsymbol{u}_{h, k, \lambda}, \boldsymbol{v}_{h, k}\right) \\
=\int_{0}^{T}\left(\rho_{h, k, \lambda} \boldsymbol{f}_{k}, \boldsymbol{v}_{h, k}\right)+\frac{1}{2} \int_{0}^{T}\left(\frac{\partial}{\partial t} \widetilde{\rho}_{h, k}, \boldsymbol{u}_{h, k, \lambda} \cdot \boldsymbol{v}_{h, k}-Q_{h}\left(\boldsymbol{u}_{h, k, \lambda} \cdot \boldsymbol{v}_{h, k}\right)\right) \\
-\frac{1}{2} \int_{0}^{T}\left(\rho_{h, k, \lambda} \widehat{\boldsymbol{w}}_{h, k, \lambda}, \nabla\left(\boldsymbol{u}_{h, k, \lambda} \cdot \boldsymbol{v}_{h, k}-Q_{h}\left(\boldsymbol{u}_{h, k, \lambda} \cdot \boldsymbol{v}_{h, k}\right)\right)\right) \\
-\frac{1}{2} \int_{0}^{T}\left(\rho_{h, k, \lambda}\left(\widehat{\boldsymbol{u}}_{h, k, \lambda}-\widehat{\boldsymbol{w}}_{h, k, \lambda}\right), \nabla\left(\boldsymbol{u}_{h, k, \lambda} \cdot \boldsymbol{v}_{h, k}-Q_{h}\left(\boldsymbol{u}_{h, k, \lambda} \cdot \boldsymbol{v}_{h, k}\right)\right)\right) \\
-\frac{\lambda}{2} k \int_{0}^{T}\left(\rho_{h, k, \lambda}, \boldsymbol{u}_{h, k, \lambda} \cdot \boldsymbol{v}_{h, k}-Q_{h}\left(\boldsymbol{u}_{h, k, \lambda} \cdot \boldsymbol{v}_{h, k}\right)\right) \\
:=\int_{0}^{T}\left(\rho_{h, k, \lambda} \boldsymbol{f}_{k}, \boldsymbol{v}_{h, k}\right)+R_{1}+R_{2}+R_{3}+R_{4},
\end{array}\right.
$$

where $\boldsymbol{v}_{h, k}$ and $\widetilde{\boldsymbol{v}}_{h, k}$ are suitable approximations of a test function $\boldsymbol{v} \in C^{1}\left([0, T] ; \boldsymbol{C}_{c}^{\infty}(\Omega)\right)$.

Again, by using the estimates independent of $\lambda$ and arguing as in the continuous case [9], one can prove that

$$
\begin{gathered}
\lambda \int_{0}^{T}\left(\nabla \rho_{h, k, \lambda} \otimes \boldsymbol{u}_{h, k, \lambda}, \nabla \boldsymbol{v}_{h, k}\right) \rightarrow 0 \quad \text { as }(h, k, \lambda) \rightarrow 0, \\
\lambda \int_{0}^{T}\left(\rho_{h, k, \lambda}-\frac{\widetilde{M}+\widetilde{m}}{2}\right)\left(\nabla \boldsymbol{u}_{h, k, \lambda}\right)^{t}, \nabla \boldsymbol{v}_{h, k} \rightarrow 0 \quad \text { as }(h, k, \lambda) \rightarrow 0 .
\end{gathered}
$$

Copyright $@$ by SIAM. Unauthorized reproduction of this article is prohibited. 
To finish the passage to the limit, we show only that the residual terms $R_{i}$ vanish as $(h, k, \lambda) \rightarrow 0$. For this, we impose that the sequence of test functions $\boldsymbol{v}_{h, k}$ is bounded in $L^{\infty}\left(0, T ; \boldsymbol{W}^{1,3}(\Omega) \cap \boldsymbol{L}^{\infty}(\Omega)\right)$.

We bound $R_{1}$, thanks to estimates (ii) and (vi) of Lemma 3.2, as follows:

$$
\left.R_{1} \leq C \sum_{n=0}^{N-1}\left|\rho_{h}^{n+1}-\rho_{h}^{n}\right| h\left\|\boldsymbol{u}_{h}^{n+1}\right\|\left\|\boldsymbol{v}_{h}^{n}\right\|_{W^{1,3} \cap L^{\infty}} \leq C \frac{h}{k} \rightarrow 0 \quad \text { (thanks to }\left(\mathrm{S}^{\prime}\right)\right) .
$$

By integrating by parts $R_{2}$,

$$
\begin{aligned}
R_{2}= & -\int_{0}^{T}\left(\widehat{\boldsymbol{u}}_{h, k, \lambda} \cdot \nabla \rho_{h, k, \lambda}, \boldsymbol{u}_{h, k, \lambda} \cdot \boldsymbol{v}_{h, k}-Q_{h}\left(\boldsymbol{u}_{h, k, \lambda} \cdot \boldsymbol{v}_{h, k}\right)\right) \\
& -\int_{0}^{T}\left(\nabla \cdot \widehat{\boldsymbol{u}}_{h, k, \lambda} \rho_{h, k, \lambda}, \boldsymbol{u}_{h, k, \lambda} \cdot \boldsymbol{v}_{h, k}-Q_{h}\left(\boldsymbol{u}_{h, k, \lambda} \cdot \boldsymbol{v}_{h, k}\right)\right):=R_{2}^{1}+R_{2}^{2} .
\end{aligned}
$$

By using the (duality) result of the Aubin-Nitsche type $\left|u-Q_{h} u\right| \leq C h\|u\|_{H^{1}(\Omega)}$, the first term of $R_{2}$ can be estimated as follows:

$$
\begin{aligned}
R_{2}^{1} & \leq \int_{0}^{T}\left\|\widehat{\boldsymbol{u}}_{h, k, \lambda}\right\|\left\|\nabla \rho_{h, k, \lambda}\right\|_{L^{3}(\Omega)} h\left\|\boldsymbol{u}_{h, k, \lambda}\right\|\left\|\boldsymbol{v}_{h, k}\right\|_{W^{1, \infty}(\Omega)} \\
& \leq C \frac{h}{k^{1 / 4} \lambda^{3 / 4}}\left\|\widehat{\boldsymbol{u}}_{h, k, \lambda}\right\|_{L^{2}\left(0, T ; H_{0}^{1}(\Omega) \lambda^{3 / 4}\right.} k^{1 / 4}\left\|\nabla \rho_{h, k, \lambda}\right\|_{L^{\infty}\left(0, T ; L^{3}(\Omega)\right)} \\
& \left.\leq C \frac{h}{k_{h, k, \lambda} \|_{L^{2}\left(0, T ; H_{0}^{1}(\Omega)\right)}} \lambda^{3 / 4}\left\|\nabla \rho_{h, k, \lambda}\right\|_{L^{4}\left(0, T ; L^{3}(\Omega)\right)} \leq C \frac{h}{k^{1 / 4} \lambda^{3 / 4}} \rightarrow 0 \quad \text { (thanks to }\left(\mathrm{S}^{\prime}\right)\right) .
\end{aligned}
$$

The convergence to zero of the other term $R_{2}^{2}$ can be made in a similar way.

The term $R_{3}$ is handled as follows:

$$
\begin{aligned}
R_{3} & \leq \int_{0}^{T}\left\|\rho_{h, k, \lambda}\right\|_{L^{\infty}(\Omega)}\left|\widehat{\boldsymbol{u}}_{h, k, \lambda}-\widehat{\boldsymbol{w}}_{h, k, \lambda} \| \nabla\left(\boldsymbol{u}_{h, k, \lambda} \cdot \boldsymbol{v}_{h, k}-Q_{h}\left(\boldsymbol{u}_{h, k, \lambda} \cdot \boldsymbol{v}_{h, k}\right)\right)\right| \\
& \leq C h \int_{0}^{T}\left\|\widehat{\boldsymbol{u}}_{h, k, \lambda}-\widehat{\boldsymbol{w}}_{h, k, \lambda}\right\|\left\|\boldsymbol{u}_{h, k, \lambda}\right\|\left\|\boldsymbol{v}_{h, k}\right\|_{W^{1,3}(\Omega) \cap L^{\infty}(\Omega)} \leq C h \rightarrow 0,
\end{aligned}
$$

where we have used (3.8) and the stability property of $Q_{h}$ in the $H^{1}$-norm.

Finally, the convergence to zero of $R_{4}$ is easy to deduce. This concludes the proof of Theorem 1.2.

Remark 8.5. The asymptotic behavior as $\lambda$ goes to zero of the scheme (7.2)-(7.5) (see Remark 7.2), associated to a problem with density-dependent diffusion, remains as an open problem. In fact, when $\lambda \rightarrow 0$, both diffusion coefficients (viscosity and mass diffusion) vanish. Therefore, we find a viscosity-vanishing problem, which is an open problem even in the continuous case.

Appendix A: Proof of Lemma 3.4. We consider the following auxiliary semidiscrete scheme: Find $\rho^{n+1} \in H^{2}(\Omega)$ as the solution of the problem:

$$
\frac{\rho^{n+1}-\rho^{n}}{k}+\boldsymbol{w}_{h}^{n} \cdot \nabla T_{m}^{M} \rho^{n+1}-\lambda \Delta \rho^{n+1}=0 \quad \text { in } \Omega,\left.\quad \frac{\partial \rho^{n+1}}{\partial \boldsymbol{n}}\right|_{\partial \Omega}=0
$$

where

$$
T_{m}^{M} \rho^{n+1}(\boldsymbol{x}, t)=\left\{\begin{array}{lll}
\rho^{n+1}(\boldsymbol{x}, t) & \text { if } & \rho^{n+1}(\boldsymbol{x}, t) \in[m, M] \\
m & \text { if } & \rho^{n+1}(\boldsymbol{x}, t)<m \\
M & \text { if } & \rho^{n+1}(\boldsymbol{x}, t)>M
\end{array}\right.
$$

Copyright $@$ by SIAM. Unauthorized reproduction of this article is prohibited. 
Lemma A.1. Problem (A.1) has a unique solution.

Proof. Let $R: H^{1}(\Omega) \rightarrow H^{1}(\Omega)$ be defined by $R w=v$, where $v$ is the weak solution of the elliptic problem:

$$
\frac{v}{k}-\lambda \Delta v=-\boldsymbol{w}_{h}^{n} \cdot \nabla T_{m}^{M} w+\frac{1}{k} \rho^{n} \text { in } \Omega,\left.\quad \frac{\partial v}{\partial \boldsymbol{n}}\right|_{\partial \Omega}=0 .
$$

Notice that $T_{m}^{M} w$ is defined a.e. in $Q$. In addition, as $-\boldsymbol{w}_{h}^{n} \cdot \nabla T_{m}^{M} w+\frac{1}{k} \rho^{n} \in H^{1}(\Omega)^{\prime}$, $R$ is well-defined, that is, there exists a unique $v \in H^{1}(\Omega)$ such that $R w=v$.

By using $v$ as a test function in (A.2) and integrating by parts, we arrive at

$$
\frac{1}{k}|v|^{2}+\lambda|\nabla v|^{2} \leq\left|\nabla \cdot \boldsymbol{w}_{h}^{n}\right|\left\|T_{m}^{M} w\right\|_{L^{\infty}(\Omega)}|v|+\left|\boldsymbol{w}_{h}^{n}\right|\left\|T_{m}^{M} w\right\|_{L^{\infty}(\Omega)}|\nabla v|+\frac{1}{k}\left|\rho^{n}\right||v| .
$$

Since $\left\|T_{m}^{M} w\right\|_{L^{\infty}(\Omega)}=M$, then $\|v\|_{H^{1}(\Omega)} \leq C$, with $C>0$ a constant independent of $w$. Therefore, by taking any $r \geq C$, one has that if $\|w\|_{H^{1}(\Omega)} \leq r$, then $\|R w\|_{H^{1}(\Omega)} \leq$ $r$. To apply Schauder's fixed point theorem we have to prove that $R: H^{1}(\Omega) \rightarrow H^{1}(\Omega)$ is continuous and compact. For this, it suffices to demonstrate that

$$
\text { if } \quad w_{l} \rightarrow w \text { in } H^{1}(\Omega), \quad \text { then } \quad R w_{l} \rightarrow R w \text { in } H^{1}(\Omega) \text { as } l \rightarrow \infty .
$$

Indeed, from $w_{l} \rightarrow w$ in $H^{1}(\Omega)$, it holds by compactness that $w_{l} \rightarrow w$ in $L^{2}(\Omega)$. Therefore, there exists a subsequence, that to simplify notation is denoted in the same way, such that $w_{l} \rightarrow w$ a.e. in $\Omega$. By virtue of dominated convergence theorem, we have

$$
\boldsymbol{w}_{h}^{n} \cdot \nabla T_{m}^{M} w_{l}+\frac{1}{k} \rho^{n} \longrightarrow \boldsymbol{w}_{h}^{n} \cdot \nabla T_{m}^{M} w+\frac{1}{k} \rho^{n} \text { in } H^{1}(\Omega)^{\prime} \text { as } l \rightarrow \infty .
$$

On the other hand, the mapping $h \in H^{1}(\Omega)^{\prime} \rightarrow z \in H^{1}(\Omega)$, where $z$ is the solution

$$
\frac{1}{k}(z, \bar{z})-\lambda(\nabla z, \nabla \bar{z})=\langle h, \bar{z}\rangle_{H^{1}(\Omega)^{\prime} H^{1}(\Omega)} \quad \forall \bar{z} \in H^{1}(\Omega),
$$

is linear and continuous (by the Lax-Milgram theorem). Then from (A.3) we have $R w_{l} \rightarrow R w$ in $H^{1}(\Omega)$.

In conclusion, $\left.R\right|_{\bar{B}(0, r)}: \bar{B}(0, r) \rightarrow \bar{B}(0, r)$ is continuous and compact. By Schauder's fixed point theorem, we have the desired result after a regularity result for elliptic equations.

THEOREM A.2. The solution of problem (A.1) verifies the maximum principle; i.e., if $m \leq \rho^{0} \leq M$, then $m \leq \rho^{n} \leq M$ for each $n \geq 1$.

Proof. Let us first see that if $\rho^{n} \leq M$, then $\rho^{n+1} \leq M$. By multiplying (A.1) by $\left(\rho^{n+1}-M\right)_{+}$and integrating over $\Omega$ (we denote $f(x)_{+}=\left\{\begin{array}{ll}f(x) & \text { if } f(x)>0, \\ 0 & \text { if } f(x) \leq 0,\end{array}\right.$ ), this gives us

$$
\begin{aligned}
& \left(\frac{\rho^{n+1}-\rho^{n}}{k},\left(\rho^{n+1}-M\right)_{+}\right)+\left(\boldsymbol{w}_{h}^{n} \cdot \nabla T_{m}^{M} \rho^{n+1},\left(\rho^{n+1}-M\right)_{+}\right) \\
& +\lambda\left(\nabla \rho^{n+1}, \nabla\left(\rho^{n+1}-M\right)_{+}\right)=0 .
\end{aligned}
$$

By using properties of the positive part function and the fact that $T_{m}^{M} \rho^{n+1}=M$ as $\left(\rho^{n+1}-M\right)_{+} \neq 0$, we rewrite it as

$$
\left(\frac{\rho^{n+1}-\rho^{n}}{k},\left(\rho^{n+1}-M\right)_{+}\right)+\lambda\left(\nabla\left(\rho^{n+1}-M\right)_{+}, \nabla\left(\rho^{n+1}-M\right)_{+}\right)=0 .
$$

Copyright $@$ by SIAM. Unauthorized reproduction of this article is prohibited. 
Therefore, we deduce from (A.4) that $\left(\rho^{n+1}-\rho^{n},\left(\rho^{n+1}-M\right)_{+}\right) \leq 0$. By adding and subtracting $M$, one arrives at $\left(\left(\rho^{n+1}-M\right)-\left(\rho^{n}-M\right),\left(\rho^{n+1}-M\right)_{+}\right) \leq 0$. Again, by the properties of the positive part function, we obtain

$$
\left(\left(\rho^{n+1}-M\right),\left(\rho^{n+1}-M\right)_{+}\right)=\left(\left(\rho^{n+1}-M\right)_{+},\left(\rho^{n+1}-M\right)_{+}\right)=\left|\left(\rho^{n+1}-M\right)_{+}\right|^{2} .
$$

By the induction hypothesis $\rho^{n}-M \leq 0$, and then $\left(\rho^{n}-M,\left(\rho^{n+1}-M\right)_{+}\right) \leq 0$. Consequently, $\left|\left(\rho^{n+1}-M\right)_{+}\right|^{2} \leq\left(\rho^{n}-M,\left(\rho^{n+1}-M\right)_{+}\right) \leq 0$, and then $\rho^{n+1} \leq M$ holds.

The proof of the other bound - if $\rho^{n} \geq m$, then $\rho^{n+1} \geq m$ - can be obtained in the same way.

Corollary A.3. Problem (A.1) is equivalent to problem (3.9).

Proof. Since $\rho^{n+1}$ the solution of problem (A.1) verifies the maximum principle $m \leq \rho^{n+1} \leq M$, then, in particular from the definition of truncating operator $T_{m}^{M}$, one has $T_{m}^{M} \rho^{n+1}=\rho^{n+1}$, and problem (A.1) is rewritten as problem (3.9).

From the uniqueness of the solution of problem (3.9), we have, in particular, that problem (3.9) verifies the maximum principle

$$
0<m \leq \rho^{n}(\boldsymbol{x}) \leq M \quad \forall \boldsymbol{x} \in \Omega \quad \forall n .
$$

Therefore, to finish the proof of Lemma 3.4, it remains to prove strong estimates (independent of $\lambda, h, k)$ for the $\rho^{n+1}$ solution of (3.9). For this, it will be fundamental to use the pointwise estimates $m \leq \rho^{n+1} \leq M$ in $Q$.

We define $\eta^{n+1}=\rho^{n+1}-\frac{1}{|\Omega|} \int_{\Omega} \rho^{n+1}$, and hence $\frac{1}{|\Omega|} \int_{\Omega} \eta^{n+1}=0$. Then the

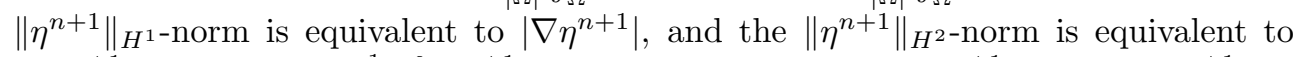
$\left|\Delta \eta^{n+1}\right|$. Since $m \leq \frac{1}{|\Omega|} \int_{\Omega} \rho^{n+1} \leq M$, the estimates for $\left\|\eta^{n+1}\right\|_{H^{1}}$ and $\left\|\eta^{n+1}\right\|_{H^{2}}$ imply estimates for $\left\|\rho^{n+1}\right\|_{H^{1}}$ and $\left\|\rho^{n+1}\right\|_{H^{2}}$, respectively.

With this definition of $\eta^{n+1}$, problem (3.9) can be rewritten as:

$$
\frac{\eta^{n+1}-\left(\rho^{n}-\frac{1}{|\Omega|} \int_{\Omega} \rho^{n+1}\right)}{k}+\boldsymbol{w}_{h}^{n} \cdot \nabla \eta^{n+1}-\lambda \Delta \eta^{n+1}=0 \text { in } \Omega,\left.\quad \frac{\partial \eta^{n+1}}{\partial \boldsymbol{n}}\right|_{\partial \Omega}=0 .
$$

By multiplying by $-2 k \Delta \eta^{n+1}$, we arrive at

$$
\begin{aligned}
& \left|\nabla \eta^{n+1}\right|^{2}-\left|\nabla\left(\rho^{n}-\frac{1}{|\Omega|} \int_{\Omega} \rho^{n+1}\right)\right|^{2}+\left|\nabla\left(\rho^{n+1}-\rho^{n}\right)\right|^{2}+2 \lambda k\left|\Delta \eta^{n+1}\right|^{2} \\
& \leq 2 k\left(\boldsymbol{w}_{h}^{n} \cdot \nabla \eta^{n+1}, \Delta \eta^{n+1}\right):=K_{1} .
\end{aligned}
$$

By integrating by parts in $K_{1}$,

$$
K_{1}=-2 k\left(\nabla \boldsymbol{w}_{h}^{n}, \nabla \eta^{n+1} \otimes \nabla \eta^{n+1}\right)+k\left(\nabla \cdot \boldsymbol{w}_{h}^{n},\left|\nabla \eta^{n+1}\right|^{2}\right) .
$$

By using $\left\|\nabla \eta^{n+1}\right\|_{L^{4}(\Omega)} \leq C\left\|\eta^{n+1}\right\|_{L^{\infty}}^{1 / 2}\left|\Delta \eta^{n+1}\right|^{1 / 2} \leq C\left|\Delta \eta^{n+1}\right|^{1 / 2}$, we get

$$
K_{1} \leq C k\left\|\boldsymbol{w}_{h}^{n}\right\|\left\|\nabla \eta^{n+1}\right\|_{L^{4}(\Omega)}^{2} \leq C k\left\|\boldsymbol{w}_{h}^{n}\right\|\left|\Delta \eta^{n+1}\right| \leq \frac{C_{\varepsilon}}{\lambda} k\left\|\boldsymbol{w}_{h}^{n}\right\|^{2}+\varepsilon \lambda k\left|\Delta \eta^{n+1}\right|^{2} .
$$

Therefore,

$$
\left|\nabla \eta^{n+1}\right|^{2}-\left|\nabla\left(\rho^{n}-\frac{1}{|\Omega|} \int_{\Omega} \rho^{n+1}\right)\right|^{2}+\lambda k\left|\Delta \eta^{n+1}\right|^{2} \leq \frac{C}{\lambda} k\left\|\boldsymbol{w}_{h}^{n}\right\|^{2} .
$$

Copyright (c) by SIAM. Unauthorized reproduction of this article is prohibited. 
By using the fact that $\nabla\left(\rho^{n}-\frac{1}{|\Omega|} \int \rho^{n+1}\right)=\nabla \rho^{n}=\nabla \eta^{n}$, we have

$$
\lambda\left\|\eta^{n+1}\right\|_{H^{1}}^{2}-\lambda\left\|\eta^{n}\right\|_{H^{1}}^{2}+\lambda\left\|\rho^{n+1}-\rho^{n}\right\|_{H^{1}}^{2}+\lambda^{2} k\left\|\eta^{n+1}\right\|_{H^{2}}^{2} \leq C k\left\|\boldsymbol{w}_{h}^{n}\right\|^{2} .
$$

By summing over $n$ and applying $k \sum_{n=1}^{N}\left\|\boldsymbol{w}_{h}^{n}\right\|^{2} \leq C$, we get the following bounds:

$$
\lambda \max _{0 \leq n \leq N}\left\|\rho^{n}\right\|_{H^{1}(\Omega)}^{2} \leq C, \quad \lambda^{2} k \sum_{n=1}^{N}\left\|\rho^{n}\right\|_{H^{2}(\Omega)}^{2} \leq C,
$$

where $C>0$ is independent of $\lambda, h, k$. This concludes the proof of Lemma 3.4.

\section{REFERENCES}

[1] S. N. Antontsev, A. V. Kazhikhov, and V. N. Monakhov, Boundary Value Problems in Mechanics of Nonhomogeneous Fluids, Stud. Math. Appl. 22, North-Holland, Amsterdam, 1990.

[2] H. Berião DA Veiga, Diffusion on viscous fluids, existence and asymptotic properties of solutions, Ann. Sc. Norm. Super. Pisa, 10 (1983), pp. 341-355.

[3] S. Brenner and L. R. Scott, The Mathematical Theory of Finite Element Methods, Texts Appl. Math. 15, Springer-Verlag, Berlin, 1994.

[4] D. Bresch, E. H. Essoufi, And M. Sy, De nouveaux systèmes de type Kazhikhov-Smagulov: Modèles de propagation de polluants et de combustion à faible nombre de Mach, C. R. Acad. Sci. Paris, Série I, 335 (2002), pp. 973-978.

[5] D. Bresch, E. H. Essoufi, AND M. Sy, Effects of density dependent viscosities on multiphasic incompressible fluid models, J. Math. Fluid Mech., 9 (2007), pp. 377-397.

[6] J. Étienne And P. Saramito, A priori error estimates of the Lagrange-Galerkin method for Kazhikhov-Smagulov type systems, C. R. Math. Acad. Sci. Paris, 341 (2005), pp. 769-774.

[7] P. G. Ciarlet, The Tinite Element Method for Elliptic Problems, North-Holland, Amsterdam, 1987.

[8] V. Girault and P. A. Raviart, Finite Element Methods for Navier-Stokes Equations: Theory and Algorithms, Springer-Verlag, Berlin, 1986.

[9] F. Guillén-GonzÁlez, Sobre un modelo asintótico de difusión de masa para fluidos incompresibles, viscoso y no homogéneos, in Proceedings of the Third Catalan Days on Applied Mathematics, Lleida, Spain, 1996, pp. 103-114.

[10] F. Guillén-González, P. Damázio, And M. A. Rojas-Medar, Approach of regular solutions for incompressible fluids with mass diffusion by an interative method, J. Math. Anal. Appl., 326 (2007), pp. 468-487.

[11] F. Guillén-GonzÁlez, And J. V. GutiÉrRez-SAntacreu, Unconditional stability and convergence of fully discrete schemes for $2 D$ viscous fluids models with mass diffusion, Math. Comp., to appear.

[12] A. Kazhikhov and Sh. Smagulov, The correctness of boundary value problems in a diffusion model of an inhomogeneous fluid, Sov. Phys. Dokl., 22 (1977), pp. 249-252.

[13] C. LiU And N. J. WALkington, Convergence of numerical approximations of the incompressible Navier-Stokes equations with variable density and viscosity, SIAM J. Numer. Anal., 45 (2007), pp. 1287-1304.

[14] R. SALvi, On the existence of weak solutions of boundary-value problems in a diffusion model of an inhomogeneous liquid in regions with moving boundaries, Port. Math., 43 (1986), pp. 213-233.

[15] P. SECCHI, On the motion of viscous fluids in the presense of diffusion, SIAM J. Math. Anal., 19 (1988), pp. 22-31.

[16] P. SECCHI, On the inicial value problem for the equations of motion of viscous incompressible fluids in the presence of diffsion, Boll. Unione Mat. Ital., 6 (1982), pp. 117-1130.

[17] J. Simon, Compact sets in the Space $L^{p}(0, T ; B)$, Ann. Mat. Pura Appl., 146 (1987), pp. 65-97.

[18] R. Temam, Navier-Stokes Equations. Theory and Numerical Analysis, North-Holland, Amsterdam, 1977.

Copyright (c) by SIAM. Unauthorized reproduction of this article is prohibited. 